ARTICLE

https://doi.org/10.1038/s41467-019-09829-z

\title{
A conserved CCM complex promotes apoptosis non-autonomously by regulating zinc homeostasis
}

Eric M. Chapman ${ }^{1,2}$, Benjamin Lant ${ }^{2}$, Yota Ohashi ${ }^{1}$, Bin Yu², Michael Schertzberg ${ }^{3}$, Christopher Go ${ }^{1,4}$, Deepika Dogra ${ }^{5}$, Janne Koskimäki ${ }^{6}$, Romuald Girard ${ }^{6}$, Yan Li (i) ${ }^{7}$, Andrew G. Fraser ${ }^{1,3}$, Issam A. Awad ${ }^{6}$, Salim Abdelilah-Seyfried (1D ${ }^{5}$, Anne-Claude Gingras (iD ${ }^{1,4} \&$ W. Brent Derry (D) ${ }^{1,2}$

Apoptotic death of cells damaged by genotoxic stress requires regulatory input from surrounding tissues. The $C$. elegans scaffold protein KRI-1, ortholog of mammalian KRIT1/CCM1, permits DNA damage-induced apoptosis of cells in the germline by an unknown cell nonautonomous mechanism. We reveal that KRI-1 exists in a complex with CCM-2 in the intestine to negatively regulate the ERK-5/MAPK pathway. This allows the KLF-3 transcription factor to facilitate expression of the SLC39 zinc transporter gene zipt-2.3, which functions to sequester zinc in the intestine. Ablation of KRI-1 results in reduced zinc sequestration in the intestine, inhibition of IR-induced MPK-1/ERK1 activation, and apoptosis in the germline. Zinc localization is also perturbed in the vasculature of $\mathrm{krit}^{-1-}$ zebrafish, and SLC39 zinc transporters are mis-expressed in Cerebral Cavernous Malformations (CCM) patient tissues. This study provides new insights into the regulation of apoptosis by crosstissue communication, and suggests a link between zinc localization and CCM disease.

\footnotetext{
${ }^{1}$ Department of Molecular Genetics, University of Toronto, Toronto, M5S 1A8 ON, Canada. ${ }^{2}$ Developmental and Stem Cell Biology Program, Peter Gilgan Centre for Research and Learning, The Hospital for Sick Children, Toronto, M5G OA4 ON, Canada. ${ }^{3}$ The Donnelly Centre for Cellular and Biomolecular Research, University of Toronto, Toronto, M5S 3E1 ON, Canada. ${ }^{4}$ Lunenfeld-Tanenbaum Research Institute at Mount Sinai Hospital, Toronto, M5G 1 X5 ON, Canada. ${ }^{5}$ Institute for Biochemistry and Biology, Potsdam University, Potsdam, 14476, Germany. ${ }^{6}$ Neurovascular Surgery Program, Section of Neurosurgery, The University of Chicago Medicine, Chicago, IL 60637, USA. 7 University of Chicago Center for Research Informatics, The University of Chicago, Chicago, IL 60637, USA. Correspondence and requests for materials should be addressed to W.B.D. (email: brent.derry@sickkids.ca)
} 
poptosis is a genetically regulated program that results in the removal of cells from a population in response to developmental cues or external stimuli ${ }^{1}$. In the nematode worm Caenorhabditis elegans, apoptosis occurs in the somatic tissue during development ${ }^{2}$, and in the germline under normal physiological conditions or in response to DNA damage ${ }^{3,4}$. Apoptosis in the soma and in response to germline DNA damage is initiated by the expression of the pro-apoptotic $\mathrm{BH} 3$-only gene egl-1 that activates a conserved signaling cascade. EGL-1 protein binds and inhibits the anti-apoptotic CED-9/BCL2, allowing for CED-4/APAF1 apoptosome formation and activation of the caspase CED- $3^{5}$. Excessive DNA damage in the germline as a result of ionizing radiation (IR), activates the p53-like transcription factor CEP-1 $1^{6,7}$, which induces expression of the BH3-only gene egl-1 to stimulate apoptosis ${ }^{8}$. In addition, the ERK1/2 homologue MPK-1 cooperates with the CEP-1 pathway ${ }^{9}$ and can also function in parallel ${ }^{10,11}$ to promote IR-induced apoptosis.

Previously, we found that KRI-1 is required in the soma to promote IR-induced germline apoptosis in a cell nonautonomous manner, independent of CEP- $1 / \mathrm{p} 53^{12}$. KRI- 1 is the ortholog of the human scaffolding protein KRIT1/CCM1, which is frequently mutated in familial occurrences of the vascular disease cerebral cavernous malformations (CCM) that can lead to strokes and seizures ${ }^{13}$. Since KRI-1 is expressed in the soma, but not the germline ${ }^{12,14}$ we wondered how this scaffold nonautonomously promotes apoptosis of damaged germ cells.

In this study, we define the apoptotic pathway downstream of KRI-1 by conducting a forward genetic mutagenesis screen to restore IR-induced apoptosis in kri-1 mutant worms. This screen uncovers a conserved ERK-5/MAPK signaling pathway and the KLF-3 transcription factor ${ }^{15,16}$. Proteomic analysis of KRI-1 binding partners identifies the previously uncharacterized protein K07A9.3, which is the ortholog of human CCM2 and a regulator of the ERK-5 pathway. Transcriptome analysis in kri-1 mutant worms reveals that the zinc transporter gene zipt-2.3 is regulated by KLF-3 and required for intestinal zinc storage. This permits MPK-1 phosphorylation in the germline to promote apoptosis. By understanding the conserved KRTI1/CCM1 signaling network it will be possible to identify novel therapeutics for a condition that currently relies on invasive surgery for treatment ${ }^{17}$.

\section{Results}

KRI-1 regulates MPK-1 to promote IR-induced apoptosis. Given that the ERK1 homologue MPK-1 is necessary in the pachytene region of the germline for IR-induced apoptosis ${ }^{9,10}$, we wondered if germline MPK-1 might be regulated by KRI-1. To test this, germlines were isolated from wild type and kri-1 (ok1251) null worms ${ }^{12,14}$ and immunostained with an antibody that recognizes the active di-phosphorylated (dp-) MPK- $1^{18}$. While levels of dp-MPK-1 were similar in wild type and kri-1 (ok1251) animals in non-irradiated conditions, kri-1(ok1251) worms had reduced levels of IR-induced dp-MPK-1 in the pachytene region compared to wild type animals (Fig. 1a). This indicates that KRI-1 in the soma is required for the full activation of MPK-1 in the germline. To determine whether increasing MPK-1 activation can restore apoptosis in kri-1(ok1251) mutants, we ablated two different negative regulators of MPK-1. First, we crossed a loss of function mutation $(z h 10)$ of the MPK-1 germline phosphatase lip- $1^{9}$ into $k r i-1$ (ok1251) mutants and found that the kri-1(ok1251); lip-1(zh10) double mutants had levels of apoptosis similar to lip-1(zh15) single mutants (Fig. 1b). Knock-down of the MPK-1 negative regulator gla-3 $3^{19}$ by RNA $i$ in $k r i-1(o k 1251)$ mutants also restored IR-induced apoptosis to similar levels as knocking-down gla-3 in wild type animals (Supplementary Fig. 1A). To determine where $k r i-1$ intersects the MAPK pathway, we crossed kri-1(ok1251) worms with a strain harboring a let-60/ Ras gain-of-function mutation ( $\mathrm{ga} 89$ ). This allele causes hyper-

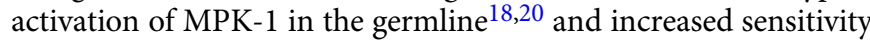
to IR-induced apoptosis ${ }^{9}$. The levels of apoptosis in the kri-1 (ok1251); let-60(ga89) double mutant was not as high as let-60 (ga89) single mutants (Supplementary Fig. 1B), indicating that KRI-1 promotes the activation of MPK-1 either downstream or independently of LET-60/Ras (Fig. 1c).

The ERK-5 pathway and KLF-3 function downstream of KRI-1. To identify genes that function downstream of kri-1, we performed a forward genetic suppressor screen using the mutagen ethyl methanesulfonate (EMS). Since scoring apoptosis in single worms under a compound light microscope is rate-limiting, a rapid screening method was required. We therefore exploited the hypersensitivity of kri-1(ok1251) mutants to starvation stress in order to select for EMS-induced mutations that suppress this phenotype (Fig. 2a), reasoning that these suppressors might also restore IR-induced germline apoptosis. From an initial selection screen involving approximately 1,000,000 haploid genomes, we isolated 300 second generation (F2) candidate suppressors that survived this period of diapause arrest (Supplementary Fig. 2A). Clonal populations from these survivors were established, and 13 of these suppressor strains isolated from unique populations exhibited a complete restoration of IR-induced apoptosis (Fig. 2b). We then performed whole genome sequencing to identify the EMS-induced mutations in the 13 kri-1(ok1251) suppressor strains. Dominance tests revealed that 12 strains had recessive mutations, while one strain harbored a dominant mutation (Supplementary Fig. 2B). Strikingly, the 12 recessive strains had non-synonymous mutations within genes that comprise the ERK-5/MAPK pathway (Fig. 2c, e). In this conserved pathway, the MAP kinase kinase kinase MEKK3 phosphorylates and activates the MAP kinase kinase MEK5 that then activates the MAP kinase ERK5 ${ }^{21}$. Six strains had mutations that result in single amino acid changes in Y106G6A.1, which is orthologous to human MEKK3 (Supplementary Fig. 2C). Of these six strains, two had unique point mutations, while four strains shared a third point mutation (Fig. 2c). Five strains had unique mutations in isoform "a" of the gene E02D9.1, which is orthologous to human MEK5 (Supplementary Fig. 2D). Three of these E02D9.1 mutations result in amino acid changes, one alters a splice donor site and one is a single amino acid deletion resulting in a predicted frame-shift (Fig. 2c). Finally, one strain had a mutation in a splice acceptor site in $m p k-2$ (Fig. 2c), which is orthologous to human ERK5 (Supplementary Fig. 2E). We henceforth refer to Y106G6A.1 as mekk-3, E02D9.1 as mek-5.

The single $k r i-1$ suppressor strain with the dominant mutation (Supplementary Fig. 2B) had a non-synonymous nucleotide change in klf-3, which encodes a "Group 2-like" KLF transcription factor ${ }^{22}$. This point mutation results in a glycine to valine substitution in the second $\mathrm{C} 2 \mathrm{H} 2$ zinc finger-DNA binding domain of KLF-3 (Fig. 2d), and is an expected gain-of-function allele since haploinsufficiency is very rare in $C$. elegans ${ }^{23}$. Since the vertebrate ERK5 pathway is known to regulate KLF transcription factors in the context of CCM disease ${ }^{16,24}$, it is likely that $k l f-3$ is part of the KRI-1/KRIT1 pathway in C. elegans.

The ERK-5 pathway and KLF-3 regulate IR-induced apoptosis. To validate that the predicted loss-of-function mutations in mekk-3, mek-5, and mpk-2 cause a restoration of apoptosis downstream of $k r i-1$, we knocked down each of these genes by RNA $i$ in wild type and $k r i-1(o k 1251)$ worms. Ablation of all three genes fully restored IR-induced apoptosis in kri-1(ok1251) animals, while no further increase was observed in wild type worms 
a
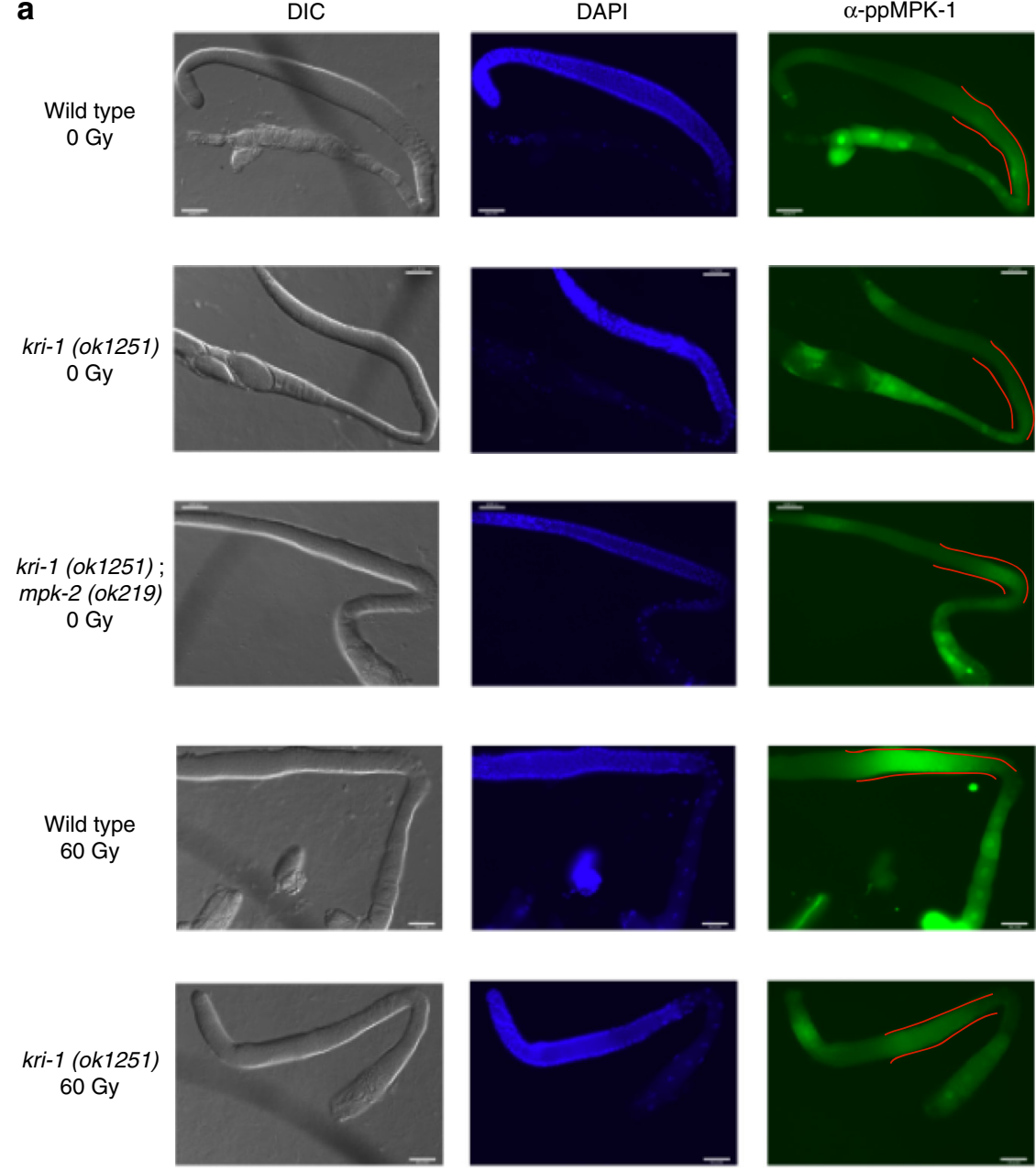

kri-1 (ok1251) 60 Gy

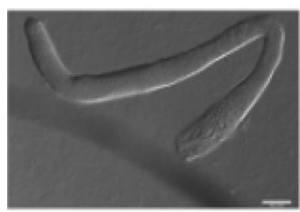

kri-1 (ok1251); mpk-2 (ok219) $60 \mathrm{~Gy}$
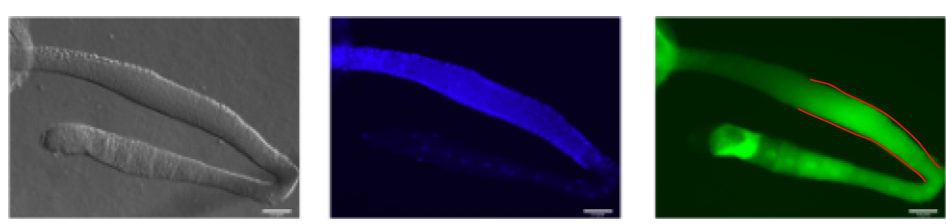

b

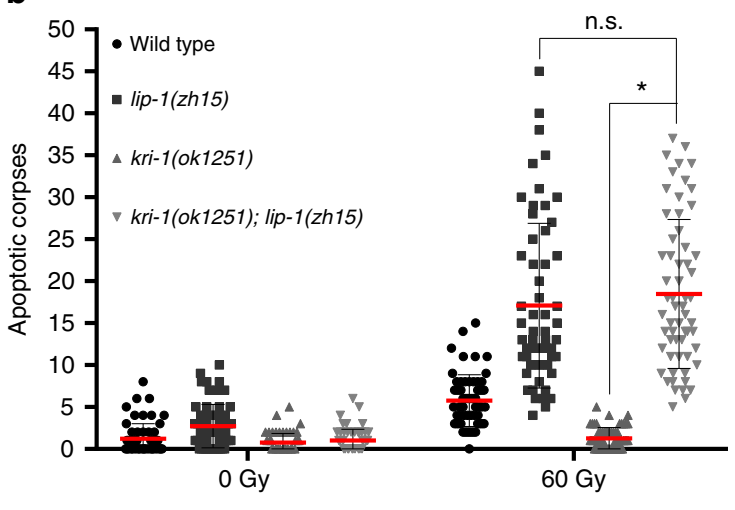

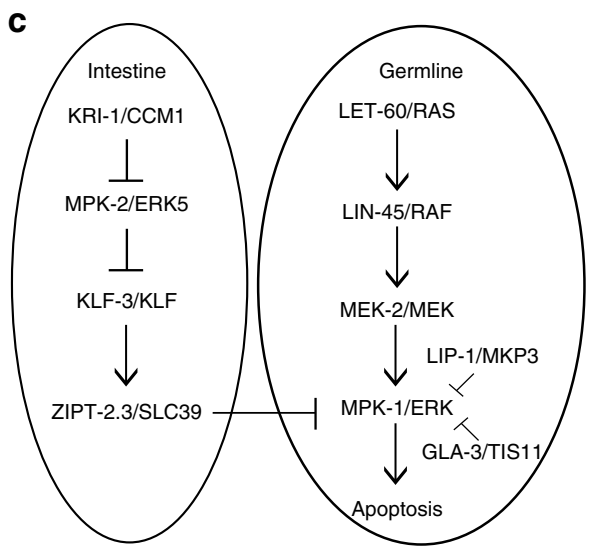

Fig. 1 KRI-1 regulates MPK-1/ERK1 in the germline. a Di-phosphorylated MPK-1 in the germlines of wild type, kri-1(ok1251), and kri-1(ok1251); mpk-2(ok219) animals is measured along the midline from the distal to proximal end. The red box on each graph corresponds to the pachytene region. Images are representative of at least 30 worms per strain, per condition ( $\times 400$ magnification). Scale bar is $25 \mu \mathrm{m}$. $\mathbf{b}$ IR-induced germline apoptosis scored in wild type, lip-1(zh15), kri-1(ok1251), and kri-1(ok1251); lip-1(zh15) animals $(n \geq 60)$. Red line is mean \pm standard deviation. ${ }^{*} P<0.05$, two-sided, unpaired $t$-test. c Schematic of intestinal KRI-1 regulating MPK-1/ERK1 in the germline 
a

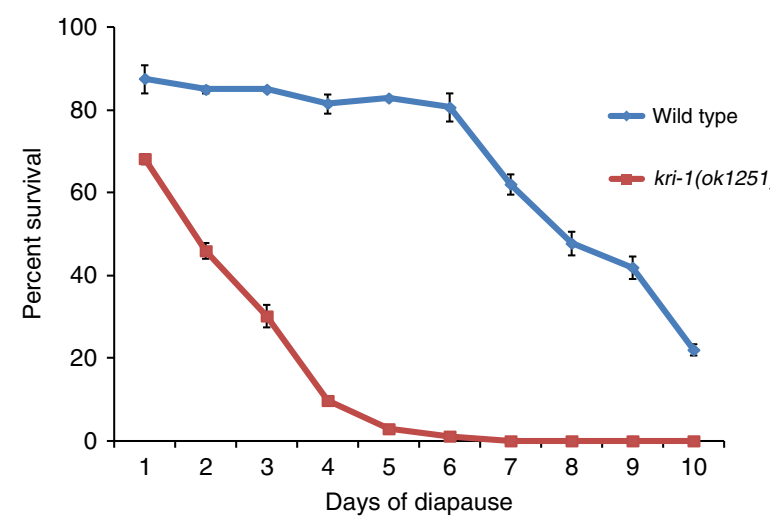

b

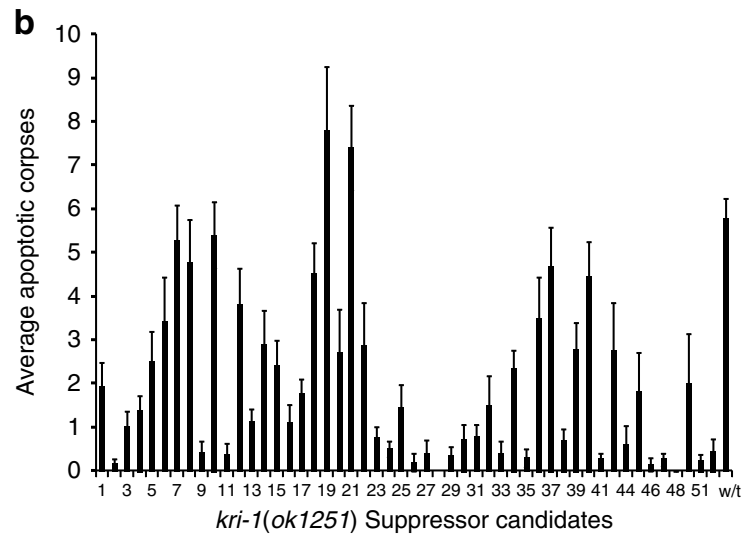

C

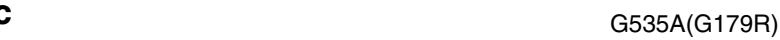
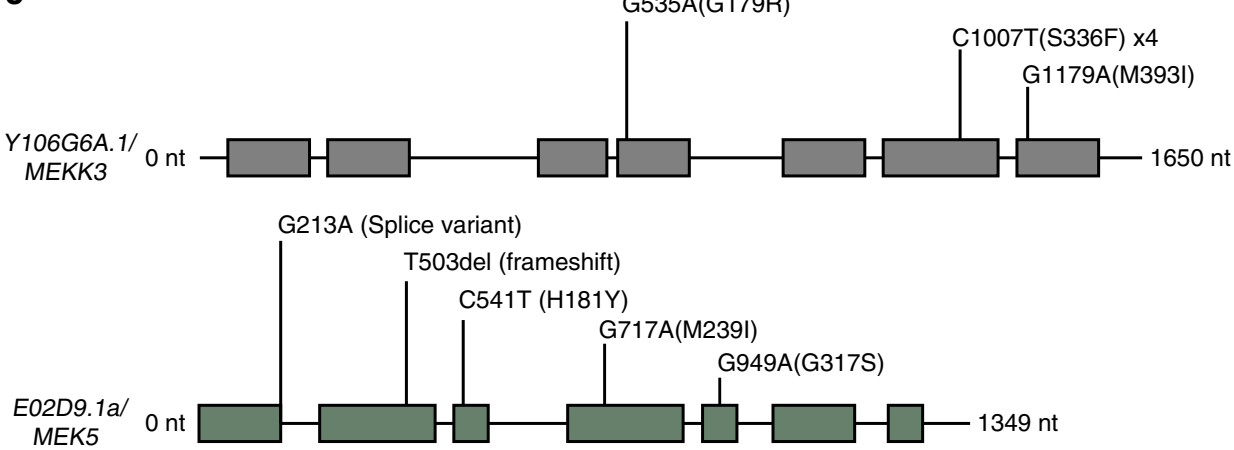

(MAP3K) MEKK3 MEK5

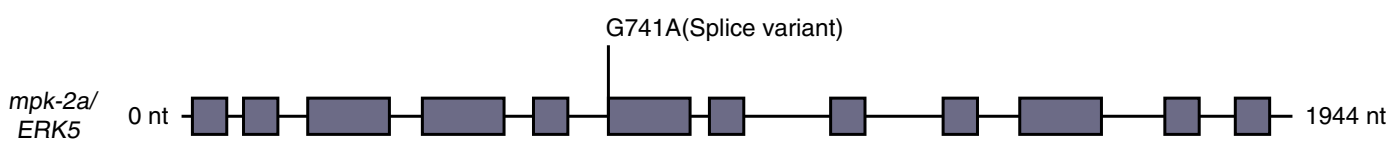

(MAP2K) MEK5

d
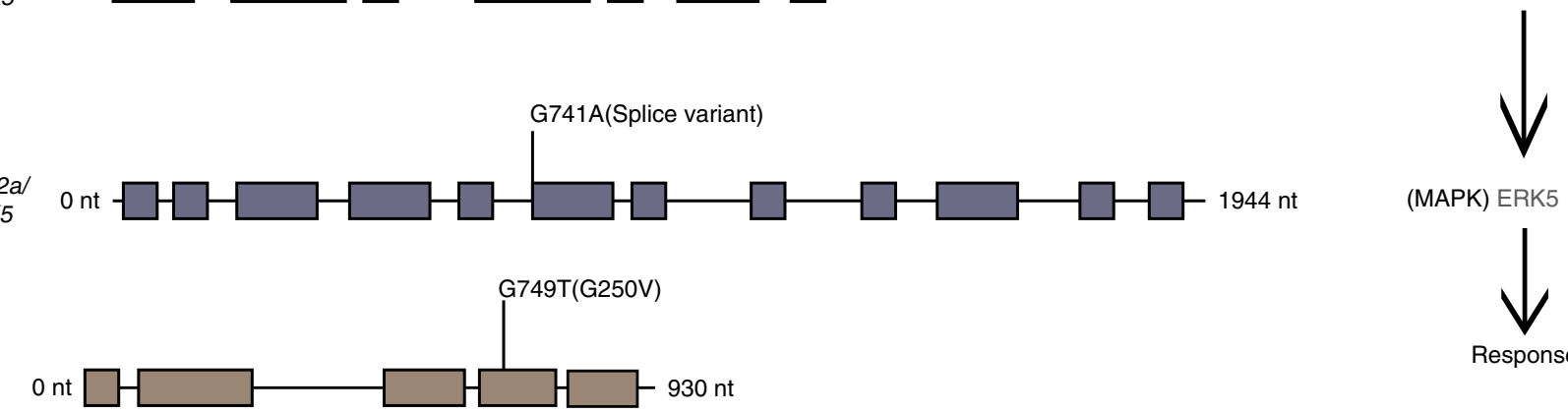

(MAPK) ERK5
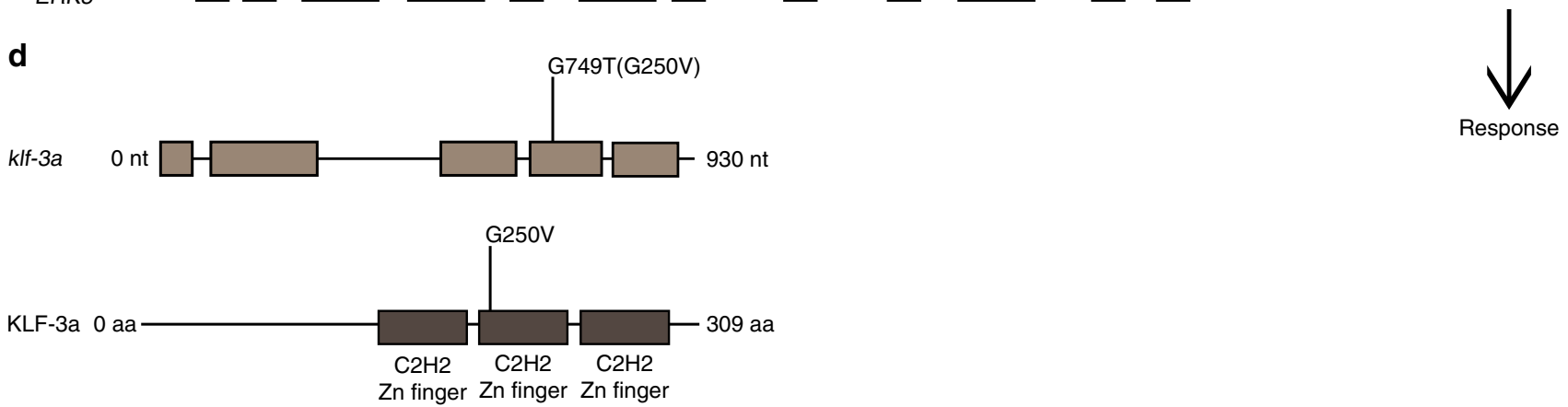

Fig. 2 A kri-1 suppressor screen identifies the ERK-5/MAPK pathway and KLF-3. a Survival of wild type and kri-1(ok1251) L1 larvae in liquid buffer (M9) without food $(n \geq 600)$. b IR-induced germline apoptosis in the progeny of kri-1(ok1251) suppressor candidates that survived 7 days of starvation ( 52 of 300 candidates depicted, $n \geq 15$ ). The black bar is the mean \pm standard deviation. c Twelve of the 13 kri-1(ok1251) suppressor candidates have recessive mutations in ERK-5/MAPK pathway genes. d The 13th kri-1(ok1251) suppressor candidate has a dominant mutation in the KLF transcription factor gene kIf3. e Depiction of the ERK-5/MAPK pathway

(Fig. 3a). To determine if this ERK-5 pathway functions as a general regulator of IR-induced apoptosis, we ablated each gene in daf-2(e1370) mutants (Fig. 3a), which are resistant to IRinduced apoptosis ${ }^{11}$ by a mechanism that is independent of $\mathrm{kri}$ $1^{12}$. Since knockdown of these genes was unable to restore apoptosis in daf-2(e1370) animals, it is likely that the ERK-5/ MAPK pathway acts downstream of KRI-1 to regulate IR-induced apoptosis. Since mekk-3 and mek-5 are very close to kri-1 on chromosome I, we decided to focus on the terminal MAPK gene, $m p k-2$ located on chromosome II for compound mutant analysis. We took advantage of the previously uncharacterized $m p k-2$ (ok219) deletion allele, which removes the 5'UTR and the first three exons of $m p k-2$ (Supplementary Fig. 3A). Using this predicted null allele, we created a kri-1(ok1251); mpk-2(ok219) double mutant and found that IR-induced dp-MPK-1 (Fig. 1a) and apoptosis (Supplementary Fig. 3B) were restored to wild type levels. These results demonstrate that resistance to IR-induced apoptosis in $k r i-1(o k 1251)$ animals is due to over-activation of the ERK-5/MAPK pathway, and that suppression of this cascade restores sensitivity. Finally, to determine if the mutation in klf-3 was responsible for restoring apoptosis in the dominant kri-1 (ok1251) suppressor strain, we generated the same klf-3 point mutation by CRISPR/Cas9, and confirmed that this new klf-3 (on34) allele functions in a dominant manner to restore 
a

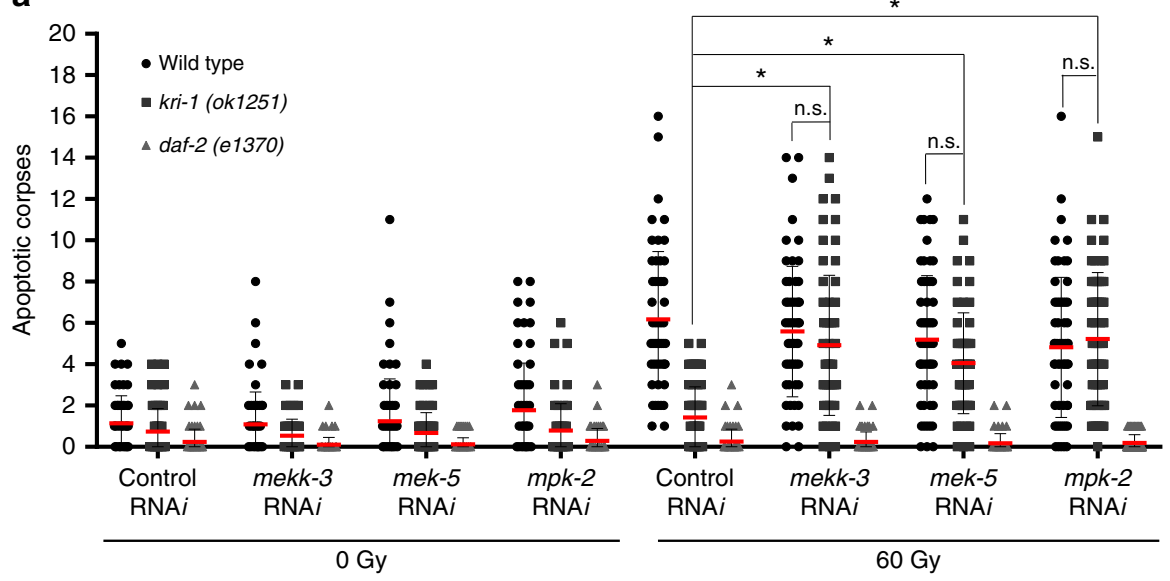

b
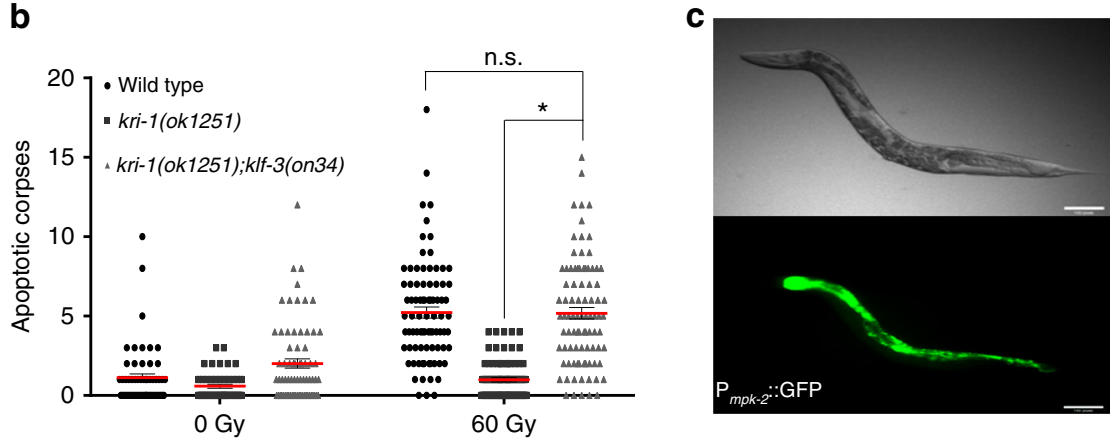

d

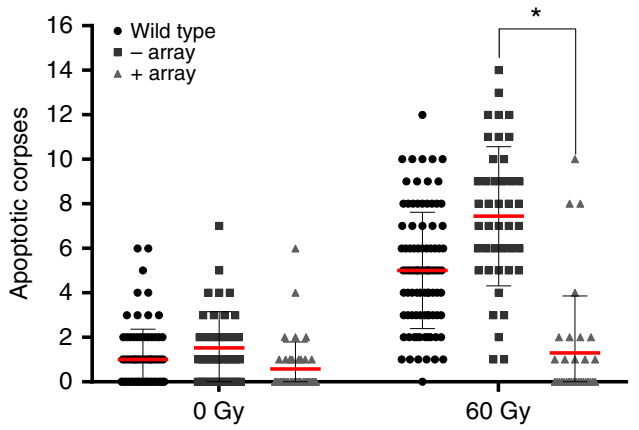

e

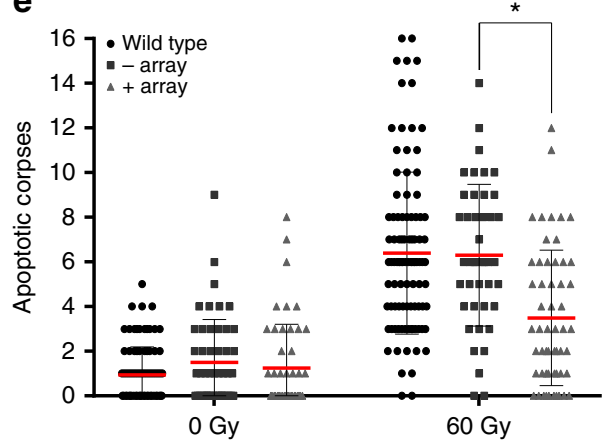

Fig. 3 MPK-2/ERK5 and KLF-3 regulate IR-induced apoptosis downstream of KRI-1. a IR-induced germline apoptosis scored after knock-down of the ERK-5/ MAPK pathway genes, mekk-3, mek-5, and mpk-2 in wild type, kri-1(ok1251) and daf-2(e1370) animals ( $n \geq 60)$. b IR-induced germline apoptosis scored in

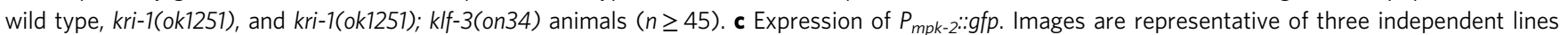

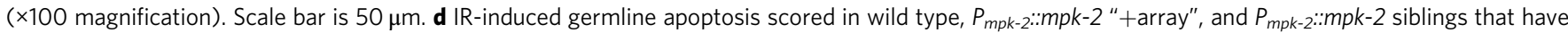

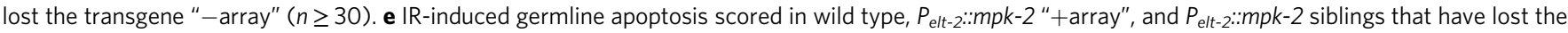
transgene "-array" $(n \geq 30)$. a-e Red line is mean \pm standard deviation. ${ }^{\star} P<0.05$, two-sided, unpaired $t$-test

IR-induced apoptosis in kri-1(ok1251) mutants (Fig. 3b; Supplementary Fig. 3C). We conclude that this mutation confers a gainof-function to KLF-3, which acts downstream of kri-1 and likely the ERK-5/MAPK cascade.

Intestinal ERK-5 regulates MPK-1 and IR-induced apoptosis. To determine where $m p k-2$ is expressed, we created a transcriptional reporter consisting of $6 \mathrm{~kb}$ of the region upstream of the $m p k-2$ start codon, fused to GFP. This construct was injected into wild type worms to form extrachromosomal (Ex) arrays ${ }^{25}$. Three independent array-containing lines express GFP in the intestine (Fig. 3c) throughout development (Supplementary Fig. 3D), similar to KRI-1::GFP ${ }^{14}$. Since our genetic analysis predicts that MPK-2 is over-activated in $k r i-1(o k 1251)$ worms, we over- expressed $m p k-2$ in wild type animals and quantified IRinduced apoptosis. Three independent Ex array-containing lines with $m p k-2$ under the control of a $6 \mathrm{~kb}$ upstream element were expressed in wild type animals, and suppression of IR-induced apoptosis was observed compared to siblings that had lost the arrays (Fig. 3d). Since Ex arrays are silenced in the germline ${ }^{26}$, this suggests that MPK-2 functions in the soma to inhibit germline apoptosis. To determine whether over-expression of $m p k-2$ specifically in the intestine can confer resistance to IRinduced germline apoptosis, we expressed $m p k-2$ under $5 \mathrm{~kb}$ of the elt-2 intestinal-specific promoter from three independent Ex arrays in wild type worms. These strains had a reduction in IRinduced apoptosis compared to siblings that had lost the array (Fig. 3e), confirming that MPK-2 regulates germline apoptosis from the intestine. In addition, kri-1(ok1251); mpk-2(ok219) 
double mutants have restored IR-induced dp-MPK-1 activation in the germline (Fig. 1a). We conclude that KRI-1 functions to suppress ERK-5/MAPK signaling in the intestine to facilitate the activation of germline MPK-1 and apoptosis in response to genotoxic stress.

CCM-2 and ICAP-1 bind KRI-1 to promote IR-induced apoptosis. Mammalian KRIT1/CCM1 regulates the ERK5/ MAPK pathway through its interaction with CCM2, which directly binds and inhibits the activation of MEKK3 $3^{27,28}$. In $C$. elegans, a CCM2 ortholog could not be identified by sequence homology-based searches, so we employed affinity purificationmass spectrometry (AP-MS) to help illuminate the mechanism by which KRI-1 regulates the ERK-5/MAPK cascade. We took advantage of a previously constructed KRI-1::GFP strain $^{14}$ that restores IR-induced apoptosis in kri-1(ok1251) mutants ${ }^{12}$. A strain that expresses actin (ACT-5) fused to GFP in the intestine $^{29}$ and wild type worms that do not express GFP were used as controls. Anti-GFP antibodies were used to immunoprecipitate whole worm lysate from irradiated and non-irradiated samples. Only four proteins were found to interact with KRI-1::GFP regardless of irradiation status (Y45F10D.10, K07A9.3, F37C4.5, and HIP-1), while HSP-17 was found to interact with KRI-1::GFP exclusively post irradiation (Fig. $4 \mathrm{a}, \mathrm{b}$ ).

F37C4.5 is an uncharacterized $C$. elegans open reading frame with no clear homologues, while HIP-1 and HSP-17 are heat shock proteins. Y45F10D.10 exhibits $25 \%$ amino acid identity to the mammalian KRIT1 binding partner, ICAP1 ${ }^{30}$ (Supplementary Fig. 4A), and consistent with this, its predicted structure is best threaded with human ICAP $1^{31}$ as a template (Phyre2 confidence score 100.0; Figure S4A). The best structural templates for threading the amino and carboxy-terminal portions of K07A9.3 are two partial structures of human CCM2, namely its PTB domain ${ }^{32}$ (Phyre2 confidence score 100.0; Supplementary Fig. 4B) and its Harmonin Homology Domain ${ }^{33}$ (Phyre2 confidence score 99.8; Supplementary Fig. 4B). These domains in human CCM2 are required to associate with KRIT1/CCM1 ${ }^{34}$ and $M E K K 3^{28}$, respectively. Due to their structural homology, we henceforth refer to Y45F10D.10 as icap-1 and K07A9.3 as ccm-2.

To determine if these KRI-1 binding partners are required for apoptosis, we knocked down each gene by RNA $i$ in wild type worms, excluding the low abundance binding partner HIP-1, and HSP-17, which might simply be responding to radiation stress. Knockdown of both $c c m-2$ and icap-1 suppressed IR-induced germline apoptosis, while F37C4.5 did not (Fig. 4c). Since CCM2 and ICAP1 bind separate NPxY/F motifs of KRIT1/CCM1 in vertebrates $^{34}$ (Supplementary Fig. 4C), we propose that the $C$. elegans KRI-1 complex contains both CCM-2 and ICAP-1, and inhibits the ERK-5 pathway through MEKK-3, as observed in vertebrates 16,24 .

zipt-2.3 expression is required for IR-induced apoptosis. To gain more insight into how MPK-2 regulates IR-induced apoptosis, we performed RNA sequencing to identify transcripts altered in $k r i-1(o k 1251)$ animals that return to wild type-like levels of expression in $k r i-1(o k 1251)$; $m p k$-2(ok219) double mutants. To do this, we treated wild type, mpk-2(ok219), kri-1 (ok1251), and kri-1(ok1251); mpk-2(ok219) animals with $60 \mathrm{~Gy}$ of IR (Fig. 4d) and identified 784 genes that were significantly increased more than two-fold and 123 genes decreased more than two-fold in kri-1(ok1251) worms (Fig. 4e; Supplementary Data 1). To determine if MPK-2 is responsible for the transcriptional changes downstream of $k r i-1$, we compared the transcriptomes of kri-1(ok1251); mpk-2(ok219) double mutant worms to kri-1 (ok1251) animals and found that 629 up-regulated, and 99 down- regulated transcripts return to wild type-like levels in the double mutants (Fig. 4e; Supplementary Data 2-3). Many of these genes are predicted to be involved in innate immunity and various stress responses (Fig. 4f). To determine which of these misregulated genes are required for IR-induced apoptosis, we conducted a targeted RNA $i$ screen using a commercially available RNA $i$ library that covers approximately $85 \%$ of the C. elegans genome. We systematically knocked down 535/629 genes that were increased two-fold or greater (Supplementary Data 4), and $87 / 99$ genes decreased two-fold or more (Supplementary Data 5). None of the up-regulated genes restored apoptosis when knocked down in kri-1(ok1251) mutants, but knockdown of two downregulated genes in wild type worms suppressed IR-induced germline apoptosis to similar levels as kri-1 mutants. These genes are pho-1, which encodes an intestinal acid phosphatase, and the SLC39 family zinc transporter zipt-2.3. We chose to focus our analysis on zipt-2.3 since high concentrations of zinc can inhibit apoptosis 35,36 , and ablation of other zinc transporters in C. elegans cause zinc storage defects ${ }^{37}$ that result in its diffusion throughout the animal ${ }^{38}$. We confirmed that zipt-2.3(ok2094) deletion mutants (Supplementary Fig. 4D) are resistant to IRinduced apoptosis (Supplementary Fig. 4E). To validate the transcriptional changes of zipt-2.3 detected by RNA sequencing we carried out qPCR to confirm its downregulation in kri-1 (ok1251) animals, which increased to wild type levels in kri-1 (ok1251); mpk-2(ok219) double mutants (Supplementary Fig. 4F). As expected, zipt-2.3 levels were restored in kri-1(ok1251); klf-3 (on34) double mutants (Supplementary Fig. 4G), indicating that transcriptional regulation of zipt-2.3 is dependent on KLF-3.

ZIPT-2.3 in the intestine promotes zinc storage. To determine the localization of ZIPT-2.3 we created a GFP translational fusion reporter with $5 \mathrm{~kb}$ of promoter region of zipt-2.3, which expresses ZIPT-2.3::GFP throughout the intestine (Fig. 5a). This expression pattern is consistent with a recent report demonstrating that the zipt-2.3 promoter drives GFP expression in the intestine ${ }^{39}$. Similar to other intestinal zinc transporters ${ }^{40}$ ZIPT-2.3::GFP localizes to gut granules (Fig. 5b), which are the main sites of zinc storage in the animal ${ }^{37}$. To determine if ZIPT-2.3::GFP expression or localization is affected by a loss of $k r i-1$, we expressed the array in kri-1(ok1251) mutants. Consistent with the RNA sequencing and $\mathrm{qPCR}$ results, we observed a strong reduction of ZIPT-2.3::GFP expression with and without IR (Fig. 5c). To determine if ZIPT-2.3 regulates zinc levels or localization, we used the dye Fluozin-3, which detects stored zinc in gut granules $^{37}$. Intriguingly, intestinal zinc signal in gut granules was lost in kri-1(ok1251) worms but restored to wild type levels in kri-1 (ok1251); mpk-2(ok219), and kri-1(ok1251); klf-3(on34) double mutants (Fig. 5d). As negative controls for gut granule zinc storage, we stained zipt-2.3(ok2094) animals as well as $p g p-2(k x 48)$ mutants that have a reduction of stored zinc due to defective gut granule formation ${ }^{41}$. Collectively, these results indicate that the KRI-1-MPK-2-KLF-3 signaling cascade in the soma ensures proper storage of zinc in the intestine.

kri-1 mutants have increased zinc in the body cavity. Next, we wondered if a reduction of stored zinc in the intestines of kri-1 (ok1251) mutants results in increased zinc levels throughout the animal. To test this hypothesis, we utilized the dye Zinpyr-1, which specifically detects zinc in the interstitial body cavity of the worm $^{38}$. Consistent with our hypothesis, we detected increased zinc in the body cavity of kri-1(ok1251) mutants, compared to wild type, kri-1(ok1251); mpk-2(ok219), and kri-1(ok1251); klf-3 (on34) worms that properly store intestinal zinc (Fig. 5d, e). As a positive control for excess body cavity zinc we stained $\mathrm{ttm}-1$ 
a

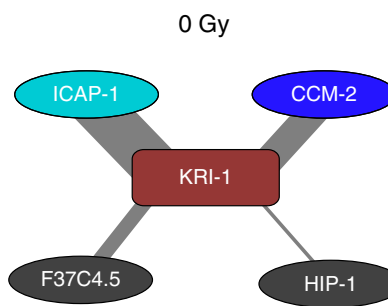

$0 . 1 \longdiv { 3 }$

Normalized spectral counts

C

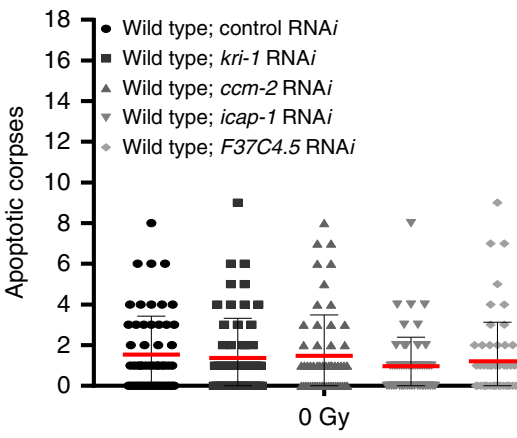

d

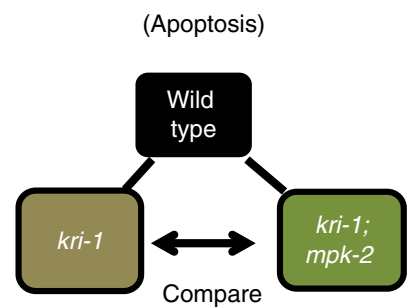

(No apoptosis)

(Apoptosis) b

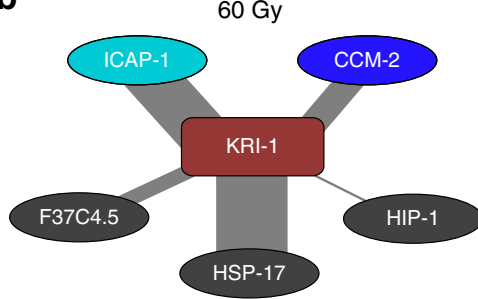

$0 . 1 \longdiv { 3 }$

e

Log2 fold change

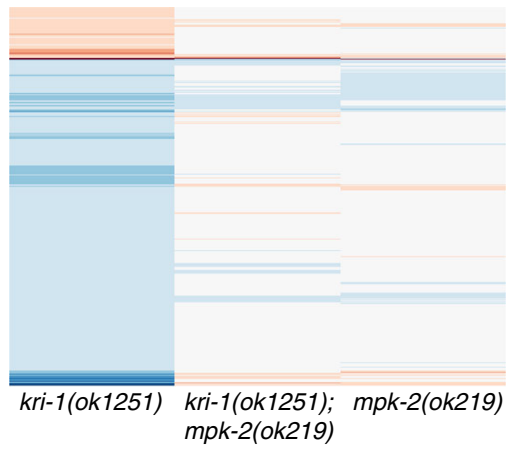

f

Genes > 2-fold increased

Innate immune response

Metabolic process

Hydrolase activity

Proteolysis

Extracellular space

Peptidase activity

Transmembrane

Neuropeptide signalling

EGF-like domain

Lipid metabolism

PP2A

Lipid transport

Cell-cell signalling

Hedgehog protein

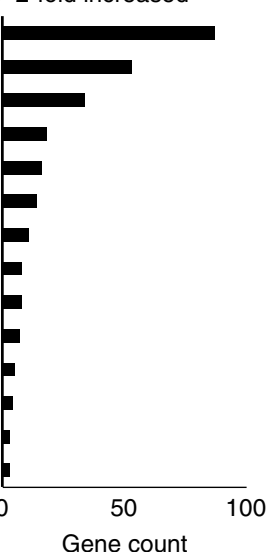

Fig. 4 KRI-1 interacts with CCM-2, ICAP-1, and regulates transcription. a, b KRI-1 interacting partners identified by AP-MS. Line thickness represents normalized spectral counts. c IR-induced germline apoptosis scored in wild type, wild type; kri-1 RNAi, wild type; ccm-2 RNAi, wild type; icap-1 RNAi, and wild type; F37C4.5 RNAi animals $(n \geq 60)$. Red line is mean \pm standard deviation. ${ }^{\star} P<0.05$, two-sided, unpaired $t$-test. $\mathbf{d}$ Schematic of mRNA sequencing. e Heat map depicting relative transcript expression in kri-1(ok1251), mpk-2(ok219), and kri-1(ok1251); mpk-2(ok219) animals compared to wild type. Red represents down-regulated transcripts, and blue represents up-regulated transcripts. f DAVID was used to group transcripts altered in kri-1(ok1251) mutants based upon gene ontology categories 


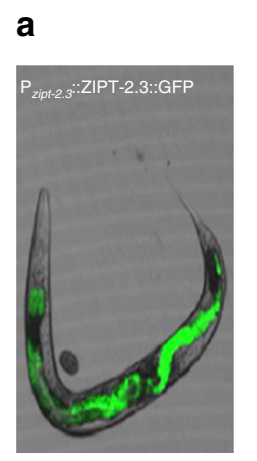

b
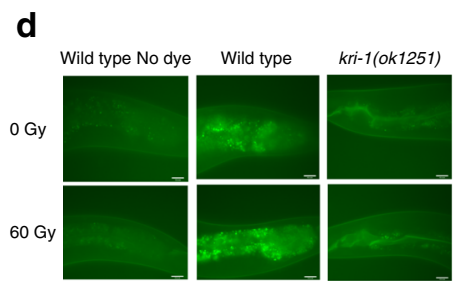

mink-

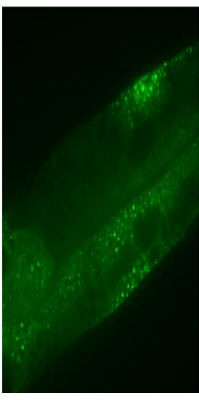

C
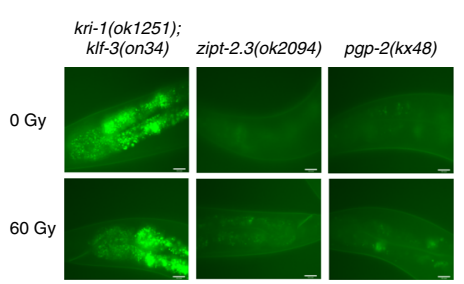

e

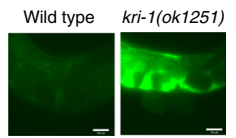

kri-1(ok1251); $k r i-1$ (ok1251);
$m p k-2$ (ok219) $\quad k l f-3(o n 34)$

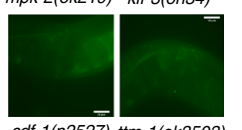

cdf-1(n2527) ttm-1(ok3503)
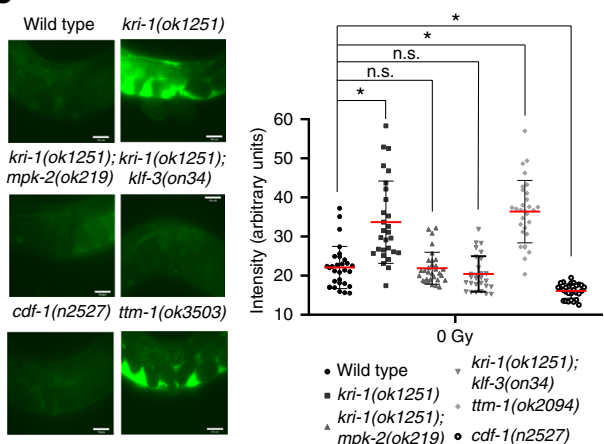

g

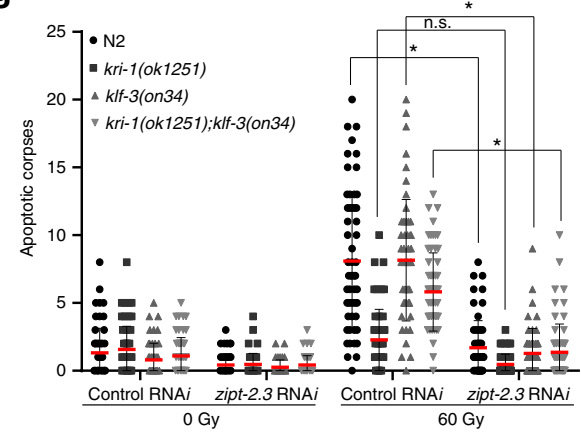

(ok3505) mutants and stained $c d f-1$ (n2527) animals for reduced body cavity zinc ${ }^{38}$. These results suggest that the failure to properly store zinc in the intestine of kri-1(ok1251) mutants leads to higher levels in the body cavity.

ZIPT-2.3 promotes IR-induced MPK-1 activation and apoptosis. To confirm that zipt-2.3 acts downstream of $m p k-2$ and klf-

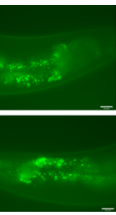

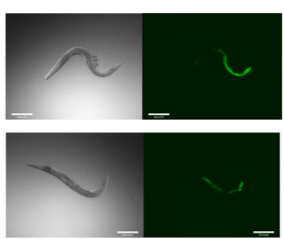
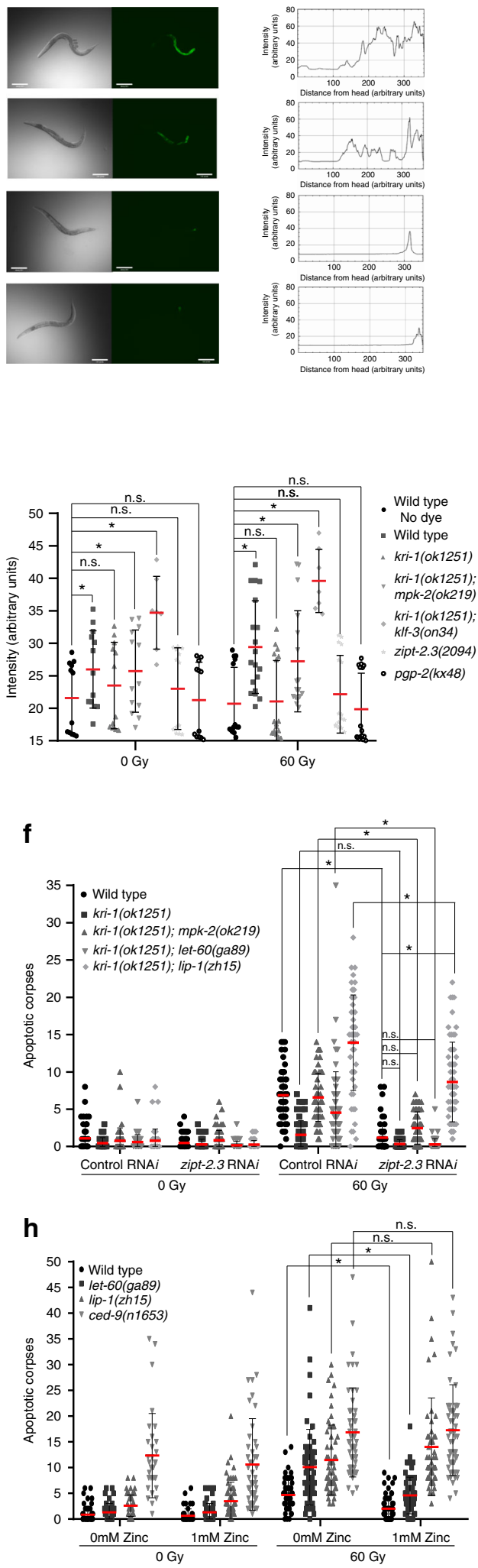

3, we knocked-down zipt-2.3 in kri-1(ok1251); mpk-2(ok219), and kri-1(ok1251); klf-3(on34) double mutant worms and observed a similar reduction in apoptosis as knocking-down zipt-2.3 in wild type animals (Fig. 5f, g). Immunostaining germlines of wild type and $k r i-1(o k 1251)$; $m p k$-2(ok219) worms for dp-MPK-1 upon zipt-2.3 RNA $i$ knock-down revealed a reduction of activated MPK-1 (Supplementary Fig. 5A), indicating that zipt-2.3 
Fig. 5 ZIPT-2.3 promotes zinc storage and IR-induced apoptosis. a, b ZIPT-2.3::GFP expression in wild type animals. Images are representative of three independent lines ( $\mathbf{a}$ is at $\times 100$ magnification and $\mathbf{b}$ is at $\times 630$ magnification). c ZIPT-2.3::GFP in wild type and kri-1(ok1251) animals is measured along the intestinal midline from head to tail. Images are representative of three independent replicates $(n \geq 15)$. Scale bar is $50 \mu \mathrm{m}$. $\mathbf{d}$ Stored intestinal zinc measured in the intestines of wild type, kri-1(ok1251), kri-1(ok1251); mpk-2(ok219), kri-1(ok1251); klf-3(on34), zipt-2.3(ok2094), and pgp-2(kx48) animals ( $n \geq 7)$. Scale bar is $25 \mu \mathrm{m}$. e Body cavity zinc measured in wild type, kri-1(ok1251), kri-1(ok1251); mpk-2(ok219), kri-1(ok1251); klf-3(on34), ttm-1(ok3505), and cdf-1(n2527) animals $(n \geq 25)$. Scale bar is $25 \mu \mathrm{m}$. f IR-induced germline apoptosis scored in wild type, kri-1(ok1251), kri-1(ok1251); mpk-2(ok219), kri-1(ok1251); let-60 (ga89) and kri-1(ok1251); lip-1(zh15) animals after knock-down of zipt-2.3 ( $n \geq 55)$. g IR-induced germline apoptosis scored in wild type, kri-1(ok1251), klf-3 (on34), and kri-1(ok1251); klf-3(on34) animals after knock-down of zipt-2.3 ( $n \geq 40$ ). h IR-induced germline apoptosis scored in wild type, let-60(ga89), lip-1 (zh15), and ced-9(n1653) animals grown on media supplemented with $1 \mathrm{mM}$ zinc $(n \geq 35)$. $\mathbf{d}$-h Red line is mean \pm standard deviation. ${ }^{\star} P<0.05$, two-sided, unpaired $t$-test

promotes apoptosis via MPK-1 activation. To establish where in the Ras/MAPK pathway zipt-2.3 functions, we knocked down zipt-2.3 in kri-1(ok1251); let-60(ga89) and kri-1(ok1251); lip-1 (zh15) mutants. Reduction of zipt-2.3 abolished the partial restoration of apoptosis in kri-1(ok1251); let-60(ga89) animals but did not suppress the restored activation in kri-1(ok1251); lip-1 (zh15) mutants (Fig. 5f). This indicates that ZIPT-2.3 is important for permitting apoptosis downstream of let-60/Ras, and upstream of $m p k-1$ (Fig. 1c).

Multiple zinc transporters regulate IR-induced apoptosis. Given that there are 28 zinc transporter genes in C. elegans ${ }^{42}$, we reasoned that there are likely zinc transporters in addition to zipt2.3 functioning in other tissues, such as the germline, to regulate IR-induced apoptosis. Of the 27 additional transporters, we first investigated zipt-7.1 since it is expressed in the germline ${ }^{43}$ and might be required for transporting zinc in this tissue. However, knockdown of zipt-7.1 by RNA $i$ did not restore IR-induced apoptosis in kri-1(ok1251) mutants, nor was IR-induced apoptosis altered in wild type animals (Supplementary Fig. 5C). Next, we systematically ablated 19/26 of the remaining C. elegans zinc transporter genes with available RNA $i$ clones, but none were able to restore apoptosis in $k r i-1(o k 1251)$ mutants (Supplementary Fig. 5B). Interestingly, ablation of Y105E8A.3, zipt-1, and zipt-13 suppressed IR-induced apoptosis in wild type animals (Supplementary Fig. 5B). It is possible that these zinc transporters also function to store zinc in the intestine.

Zinc inhibits IR-induced apoptosis. Our results thus far are consistent with a model whereby KR1-1 regulates zipt-2.3 and zinc storage to render the germline competent for apoptosis. To determine if zinc itself affects IR-induced germline apoptosis, we placed juvenile (L4 stage) nematodes on growth media containing a range of exogenous zinc concentrations $(0.1,0.5$, and $1 \mathrm{mM})$ for $24 \mathrm{~h}$. All three concentrations of zinc resulted in an inhibition of IR-induced germline apoptosis (Supplementary Fig. 6A). These animals were otherwise healthy, indicating that the concentrations of zinc tested do not affect other biological processes such as rate of growth or fertility. We also found that $4 \mathrm{~h}$ of exposure to 1 $\mathrm{mM}$ liquid zinc suppressed IR-induced apoptosis (Supplementary Fig. 6B). The reduction in IR-induced germline apoptosis by exogenous zinc is similar to kri-1(ok1251) mutants.

To determine if reducing zinc levels would result in increased IR-induced apoptosis, we incubated worms for $4 \mathrm{~h}$ in liquid buffer with the zinc chelator TPEN (N,N,N',N'-tetrakis (2-pyridinylmethyl)-1,2-ethanediamine). We chose $25 \mu \mathrm{M}$ since higher concentrations of TPEN have toxic effects ${ }^{44}$. Exposure to TPEN enhanced IR-induced apoptosis in wild type animals, and restored IR-induced apoptosis in kri-1(ok1251) mutants (Supplementary Fig. 6C). To confirm that TPEN reduces body cavity zinc, wild type animals were incubated with the Zinpyr-1 zinc dye and a reduction of fluorescence in the body cavity was observed
(Supplementary Fig. 6D). This indicates that TPEN is effective at chelating zinc, and implicates a link between body cavity zinc and IR-induced germline apoptosis.

Next, we wanted to determine zinc availability in animals by quantitative methods. For this, we used inductively coupled plasma mass spectrometry (ICP-MS ${ }^{40}$ to assess the levels of zinc in populations of wild type, kri-1(ok1251), and kri-1(ok1251); $m p k-2(o k 219)$ animals. Since we found that the levels of total zinc were the same across all three strains (Supplementary Fig. 6E), defective zinc storage in $k r i-1(o k 1251)$ mutants must result in redistribution to other tissues, as indicated by Fluozin-3 and Zinpyr-1 staining (Fig. 5d, e). To confirm that kri-1(ok1251) mutants are unable to store zinc, we incubated animals with 0.1 $\mathrm{mM}$ exogenous zinc, and analyzed zinc levels by ICP-MS. As expected, the level of zinc after exogenous exposure was lower in kri-1(ok1251) mutants compared to kri-1(ok1251); mpk-2(ok219) and wild type animals (Supplementary Fig. S6E). We conclude that kri-1(ok1251) mutants are defective in zinc storage, similar to what has been observed with $c d f-2$ mutants ${ }^{38}$.

Zinc inhibits IR-induced apoptosis upstream of MPK-1. To determine if zinc inhibits IR-induced germline apoptosis by suppressing the ERK1/MAPK pathway, we grew let-60(ga89)/Ras(gf) and lip-1(zh15) animals on $1 \mathrm{mM}$ zinc. Apoptosis was reduced in let-60(ga89) but not lip-1(zh15) mutants (Fig. 5h), indicating that zinc regulates apoptosis downstream of let-60/Ras but upstream of $m p k-1$. Since zinc has been shown to inhibit caspases ${ }^{36}$, we wondered whether this was an additional level of regulation in the germline. To test this, we grew ced-9(n1653) animals, which have enhanced caspase activation and apoptosis ${ }^{45}$ (Supplementary Fig. 5D), on $1 \mathrm{mM}$ zinc, but observed no effects (Fig. 5h). This suggests that in the germline, zinc suppresses apoptosis by inhibiting the MPK-1 MAPK pathway, but not caspase activity. Consistent with this, knockdown of zipt-2.3 does not reduce apoptosis in ced-9(n1653) mutants (Supplementary Fig. 5E).

Zinc localization and transporters are altered in CCM models. Given the conservation of the core signaling pathway downstream of KRI-1 we wondered if zinc homeostasis was similarly disrupted in vertebrates. To assess whether vascular zinc levels change in the absence of KRIT1/CCM1, we first determined the ability of Zinpyr-1 to detect zinc in developing wild type zebrafish. By 3 days post fertilization ( $\mathrm{dpf}$ ), zinc was readily detected in the posterior cardinal vein $(\mathrm{PCV})$ and caudal vein $(\mathrm{CV})$ regions of the trunk vasculature, with a similar pattern of localization by $4 \mathrm{dpf}$ (Fig. 6a). By $3 \mathrm{dpf}$ zinc signal is lost in the PCV and CV regions of krit $1 / \mathrm{ccm} 1$ mutants (Fig. 6b), suggesting that zinc localization is also regulated by KRIT1/CCM1 in vertebrates.

To investigate the specific contribution of zinc transporter regulation by KRIT1/CCM1, we assessed endothelial-specific conditional Krit1/Ccm 1 null mice using $P d g f b$ promoter driven tamoxifen-inducible Cre recombinase ${ }^{46}$, and compared RNA 
a

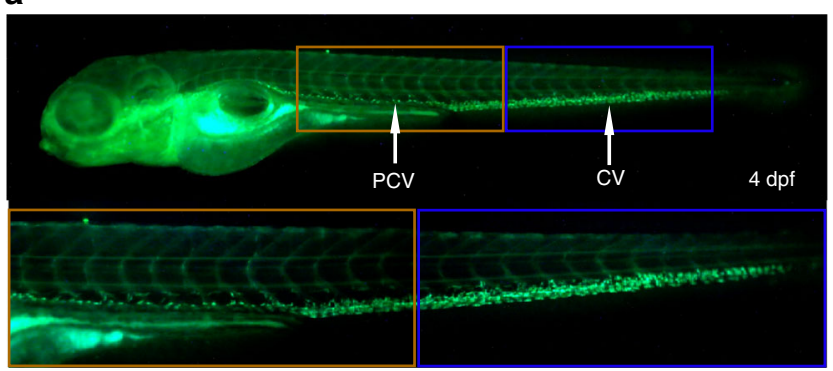

b

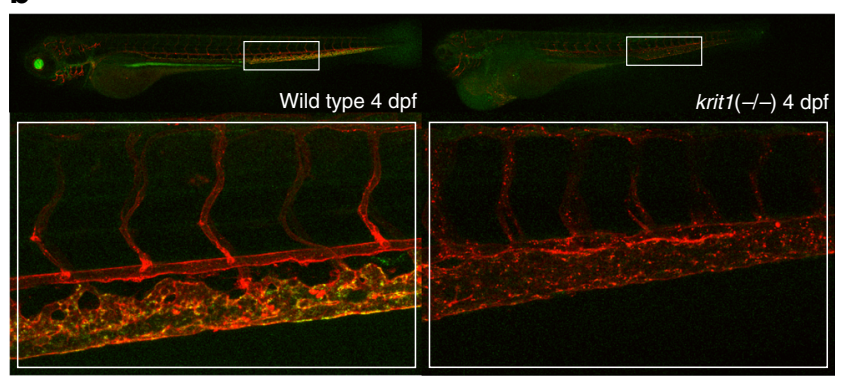

C

\begin{tabular}{lccc} 
& Fold change & $p$-Value & $\begin{array}{c}p \text {-Value } \\
\text { (FDR adjusted) }\end{array}$ \\
\cline { 2 - 4 } Human & & & \\
SLC39A10 & -3.27 & $<0.0001$ & 0.0005 \\
SLC39A11 & 3.44 & 0.002 & 0.03 \\
SLC39A3 & 2.44 & 0.01 & 0.10 \\
BMEC & & & \\
Ccm1 & & & \\
SIc39a4 & -1.56 & 0.0001 & 0.001 \\
SIc39a8 & -1.50 & 0.0003 & 0.004 \\
SIc39a3 & 1.14 & 0.02 & 0.11
\end{tabular}

Fig. 6 Zinc localization and SLC39 zinc transporter expression in CCM models. a Zinypr-1 detects zinc in the posterior cardinal vein (PCV) and caudal vein $(\mathrm{CV})$ vasculature of wild type zebrafish 4 days post fertilization (dpf). $\mathbf{b}$ Zinc localization in the PCV and CV is lost in the absence of krit1/ $\mathrm{ccm} 14 \mathrm{dpf}$. The endothelial cell vasculature is marked in red, and zinc appears green (yellow overlay). c SLC39 zinc transporter expression in CCM lesion tissue from patients (human) and in brain microvasculature endothelial tissue (BMEC) of endothelial-specific knockout mice (BMEC C $\mathrm{cm} 1$ )

from isolated brain microvascular endothelial cells (BMEC) with wild-type mice. In Krit1/Ccml mouse mutant BMECs there was a significant downregulation of Slc39a4 and Slc39a8 transcripts (Fig. 6c), and a slight upregulation of Slc39a3. To determine if the transcriptional regulation of SLC39 zinc transporters is relevant to the pathology of CCM disease, we analyzed endothelial cell (EC) RNA from five human CCM lesions (four sporadic and one familial CCM1) and compared these samples to corresponding brain tissue from three patients free of neurological disease. Transcriptome analyses showed that SLC39A10 was downregulated in ECs from human lesions compared to normal ECs from capillaries (Fig. 6c). In addition, SLC39A11 and SLCA39A3 were up-regulated in human CCM lesions (Fig. 6c). While this demonstrates that SLC39 zinc transporter genes are mis-regulated in the absence of CCM1 in C. elegans, mice, and humans, the extent to which this might contribute to CCM disease remains to be investigated.

\section{Discussion}

In this study, we set out to determine how the KRI-1 scaffold protein transmits pro-apoptosis signals from the soma (intestine) to the germline in C. elegans. By taking an unbiased forward genetic approach we uncovered three genes that comprise a canonical ERK-5/MAPK pathway, as well as the KLF-3 transcription factor, that functions in the intestine with KRI-1 to regulate apoptosis (Fig. 7). In vertebrates, the ERK5/MAPK pathway has been shown to be suppressed by the KRI-1 homologue KRIT1/CCM1 in conjunction with its binding partner CCM2. The CCM2 C-terminal Harmonin domain interacts with MEKK $3^{28}$, and ERK5 is hyperactivated downstream of MEKK3 when KRIT1 is ablated in the zebrafish myocardium ${ }^{16}$, as well as the mouse endothelium ${ }^{24}$. This over-activation of ERK5 in the mouse endothelium results in the formation of CCM lesions. Given that auto-phosphorylation of MEKK3, and subsequent activation of ERK5 is increased in the absence of CCM2 27 , it is likely that in C. elegans KRI-1 functions to attenuate ERK-5 signaling by a similar mechanism through MEKK-3. Our identification of a C. elegans CCM2 ortholog, along with the KRIT1 binding partner ortholog Y45F10D.10/ICAP1, demonstrates that the KRIT1/CCM1 signaling complex is conserved across species. In mice, endothelial-specific ablation of ERK5 results in apoptotic cell death ${ }^{48}$ and in HeLa cells ERK5 suppresses IR-induced apoptosis $^{49}$. Therefore, it is possible that the anti-apoptotic function of ERK5 signaling might contribute to the pathology of CCM disease.

In vertebrates, over-activation of ERK5 results in increased transcript levels of $K L F 2$ and $K L F 4^{15,50}$, which is predicted to contribute to CCM disease ${ }^{24}$. While $C$. elegans $k l f-3$ transcript levels were not altered in $k r i-1(o k 1251)$ animals (Supplementary Data 1), we identified a gain-of-function mutation in KLF-3 that restores IR-induced apoptosis in kri-1(ok1251) mutants. Despite the potential differences between worms and vertebrates in how ERK5 regulates KLFs, we hypothesize that the gain-of-function mutation in KLF-3 expressed in the intestine ${ }^{51}$ restores normal transcription in the absence of KRI-1. We discovered many genes that were transcriptionally altered in kri-1(ok1251) mutants, including genes affecting non-apoptotic processes such as innate immunity, suggesting that KRI-1-ERK-5-KLF-3 signaling has many other roles in the cell. For example, KRI-1 has been shown to regulate lifespan by a mechanism that is independent of apoptosis ${ }^{12,14}$. Thus, in the future it will be interesting to determine if the other biological functions of KRI-1 require these altered genes. Analysis of differentially regulated transcripts in kri-1(ok1251) mutants uncovered the SLC39 zinc transporter zipt2.3, which we confirmed to be regulated by KLF-3. The established links between zinc transporters and apoptosis ${ }^{52}$, and the possibility that zinc acts cell non-autonomously as a signaling molecule prompted us to examine the role of zinc in germline apoptosis.

Recently, zipt-2.3 was shown to be transcriptionally regulated in response to zinc stress ${ }^{39}$. Here, we observed a striking decrease of stored zinc in vesicular gut granules of $k r i-1(o k 1251)$ as well as zipt-2.3(ok2094) mutants (Fig. 5d), demonstrating that ZIPT-2.3 is dynamically regulated by KRI-1 to promote zinc storage. Zinc is required for the normal functions of approximately 3000 proteins, reinforcing the importance of this cation in cellular and developmental biology ${ }^{53}$. This is apparent from increased levels of zinc resulting in lifespan, developmental, reproductive, locomotive, and chemotaxis defects ${ }^{54}$ in C. elegans. Furthermore, zinc exposure activates stress responses in most tissues, and behavioral toxicities were found to be transferred to progeny ${ }^{54}$. Thus, $C$. elegans employs strict homeostatic mechanisms to prevent excess zinc from inducing adverse effects, such as inhibiting stressinduced apoptosis. For instance, the histidine ammonia lyase, HALY-1, regulates zinc tolerance by modulating levels of histidine $\mathrm{e}^{55}$. Since histidine functions as a zinc chelator, higher levels of this amino acid can reduce the toxic effects of excess zinc ${ }^{55}$. Inversely, reducing zinc levels in C. elegans with zinc chelators 

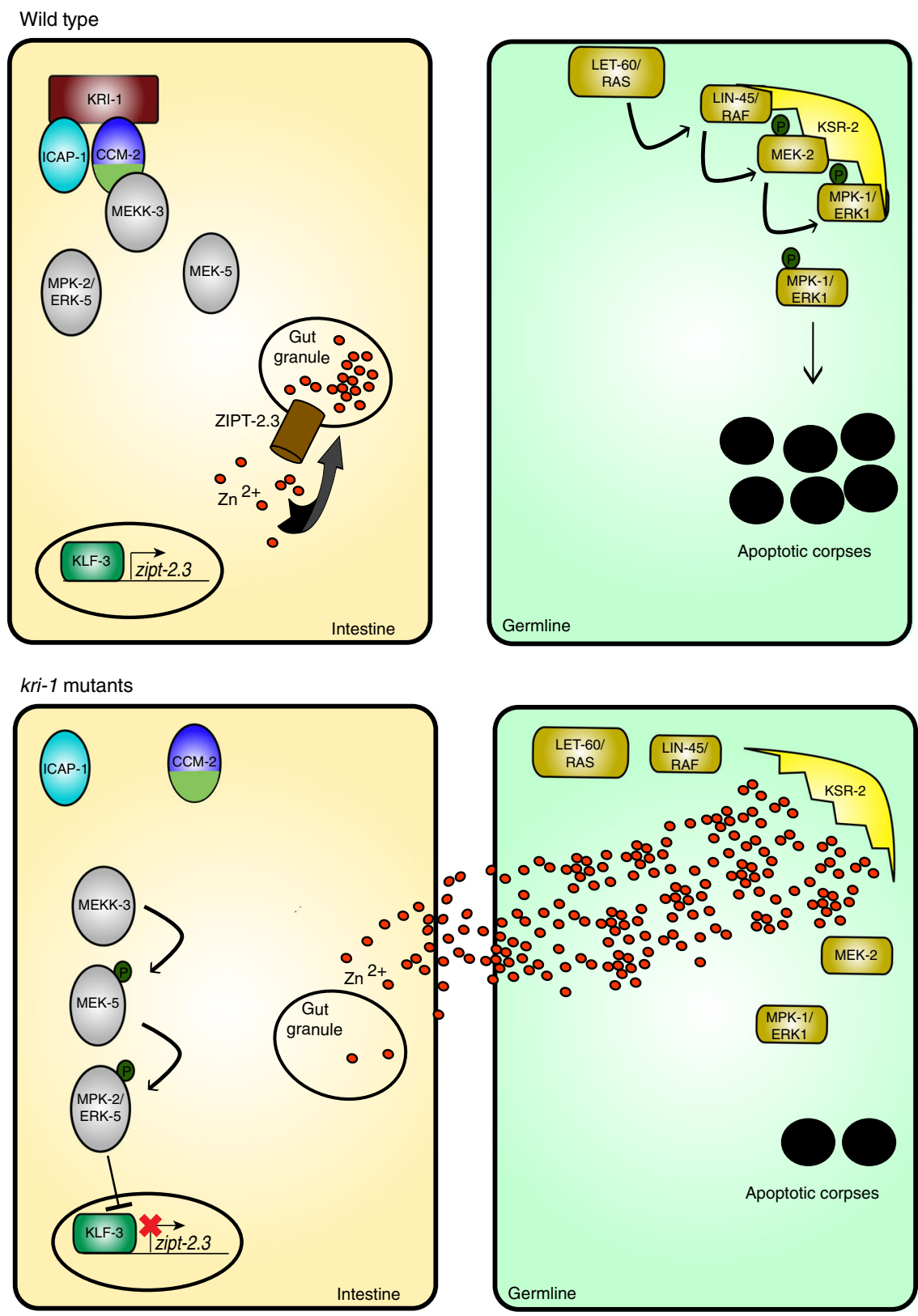

Fig. $7 \mathrm{KRI}-1$ promotes IR-induced apoptosis by regulating zinc. Schematic depicting the mechanism by which intestinal KRI-1 regulates germline MPK-1 and apoptosis. KRI-1 in complex with CCM-2 prevents the over-activation of MPK-2, allowing the transcription of zipt-2.3 by KLF-3. ZIPT-2.3 ensures the storage of zinc in the intestinal gut granules, which permits MPK-1 activation to promote apoptosis

results in increased lifespan, which is correlated with improved proteostasis $^{56}$. Collectively, this indicates that finely balanced zinc levels are critical to cellular health, which we also found to be true for apoptosis regulation. In vertebrates, zinc has been shown to prevent apoptosis by inhibiting the function of pro-apoptotic multi-BH3 domain proteins such as BAX and $\mathrm{BAK}^{35}$, and Caspase- $3^{36}$. The absence of BAX and BAK proteins in C. elegans $^{57}$ and the inability of zipt-2.3 knockdown or high zinc levels to suppress apoptosis in ced-9(n1653) animals (Fig. 5h; Supplementary Fig. 5D) indicates that zinc does not likely affect the core apoptosis pathway in this animal.

In the context of $C$. elegans vulval development, excess zinc and loss of zinc transporters have been shown to suppress the ERK1/ MAPK pathway downstream of LET-60/RAS ${ }^{58}$, and upstream of MPK $-1^{59}$. Consistent with these observations, we found that regulation of zipt-2.3 and zinc affects IR-induced germline apoptosis downstream of let-60, and upstream of $m p k-1$. We detected increased zinc throughout the body in kri-1(ok1251) animals and confirmed by ICP-MS that kri-1(ok1251) animals are unable to store zinc. Furthermore, we were able to restore IRinduced apoptosis in kri-1(ok1251) mutants by chelating nonstored zinc with TPEN. It is possible that failure to store zinc in intestinal vesicles results in leakage into the germline, where it could inhibit ERK1/MAPK signaling (Fig. 7). Since zinc has been shown to inhibit the function of $\mathrm{RAF}^{60}$ and $\mathrm{KSR}^{59}$ in C. elegans, it is possible that zinc suppresses MPK-1 activation in the germline at either of these steps in the pathway.

While we show that the KRIT1/CCM1 cascade is conserved from nematodes to humans, it remains to be determined whether zinc regulation is relevant to CCM disease in humans. Encouragingly, loss of zinc in the PCV and $\mathrm{CV}$ vasculature in $\mathrm{krit} 1 / \mathrm{ccm} 1$ mutant zebrafish suggests a conserved role for KRIT1 in zinc 
regulation in vertebrates. In zebrafish, zinc is known to affect brain acetylcholine levels, memory and motor activities ${ }^{61}$, while zinc chelation is neuroprotective in a zebrafish model of hypoxic brain injury ${ }^{62}$. These findings reveal that maintaining proper zinc levels might be important for normal brain function, and possibly to prevent the development of neurological diseases such as CCM.

Surveying zinc transporter gene regulation in $\mathrm{Krit1} / \mathrm{Ccm} 1$ mouse BMECs and patient CCM tissue revealed mis-expression of several transporters, consistent with our observations in $C$. elegans. Intriguingly, a recent report described a role for endothelial-expressed SLC39A8 in regulating mouse myocardium development by promoting proper zinc localization ${ }^{63}$. Since myocardium development is altered in a similar manner upon loss of CCM proteins in zebrafish ${ }^{15,16}$, and given that we found Slc39a8 to be down-regulated in Krit1/Ccm1 mouse BMECs (Fig. 6c), it is possible that aberrant zinc regulation plays a role in the development of CCM lesions. Future studies will be important to test this experimentally using zinc transporter mutant mouse models.

In summary, we have uncovered KRI-1 as being part of a conserved CCM complex that promotes germline apoptosis nonautonomously from the intestine. Additionally, since this core complex is conserved across species, we suggest that the discovery of KRI-1/KRIT1 linked zinc regulation, through zinc transporters, may impact the pathology of CCM disease.

\section{Methods}

Contact for reagent and resource sharing. Any queries and reagent requests should be sent to the corresponding author.

Experimental model and subject details. All nematode strains were maintained at $16^{\circ} \mathrm{C}$ and kept at $20^{\circ} \mathrm{C}$ for experimentation on NGM (nematode growth medium) agar plates with OP50 Escherichia coli as a food source, following standard procedures. N2 Bristol was used as the wild type strain and mutant strains are listed in Supplementary Table 1. HT115 E. coli was used for RNA $i$ knockdown. Zebrafish were maintained under standard conditions and strains used in this study are listed in Supplementary Table 1. Zebrafish embryos or larvae were treated with $20 \mu \mathrm{M}$ Zinpyr-1 for $30 \mathrm{~min}$, prior to imaging. The embryos were treated with 1 phenyl-2-thiourea (PTU) prior to the appearance of pigmentation. Handling of zebrafish was performed in compliance with German and Brandenburg state law, carefully monitored by the local authority for animal protection (LUVG, Brandenburg, Germany; Animal Protocol \#2347-18-2015).

RNAi feeding in $\mathbf{C}$. elegans. The RNA $i$ clones in this study were obtained from Source BioSciences, which covers approximately $85 \%$ of the C. elegans genome. Bacteria colonies were grown overnight in LB with $100 \mu \mathrm{g} / \mathrm{mL}$ ampicillin and 10 $\mu \mathrm{g} / \mathrm{mL}$ tetracycline at $37^{\circ} \mathrm{C}$ and concentrated $10 \times$ the next day. NGM plates were seeded with $100 \mu \mathrm{l}$ of the concentrated culture and synchronous L1 animals were grown at $20^{\circ} \mathrm{C}$. As a control HT115 containing the L4440 vector with the nonexpressed gene Y95B8A_84.g was used.

Germline apoptosis quantification. Worms were irradiated $24 \mathrm{~h}$ post the L 4 stage using a $\mathrm{C}^{137}$ source and apoptotic germ cells quantified $24 \mathrm{~h}$ post irradiation (60 Gy). Animals were mounted on $4 \%$ agarose pads on glass slides with $20 \mathrm{mM} \mathrm{L}$ tetramisole in M9 buffer. Apoptotic cells were scored in one germline arm by manually counting the number of raised and refractile corpses using a Leica DMRA2 system (Wetzlar, Germany) utilizing standard Differential Interference Contrast optics.

Statistical analyses. Microsoft Excel was used to determine statistical significance using a two-sided Student's $t$-test, assuming equal variance. Data was considered significant when the $P$-value was less than 0.05 . The mean (red bar) \pm standard deviation was included on all dot plots. All experiments were repeated three times, unless otherwise stated.

Imaging. All imaging with C. elegans was carried out with a Leica DMRA2 system (Wetzlar, Germany) using standard differential interference contrast optics and fluorescent channels. Fluorescence intensity was measured using ImageJ software (version 1.52a). Zebrafish embryos were imaged with a LSM 710 confocal microscope (Zeiss).
Germline dissection and p-MPK-1 staining. Young adult worms ( $24 \mathrm{~h}$ post L4) were irradiated with either 60 or 0 Gy of IR, and washed once in $1 \times$ PBS $3 \mathrm{~h}$ post $\mathrm{IR}$, and transferred to $30 \mu \mathrm{l} 1 \times \mathrm{PBS}+4 \mathrm{mM}$ Tetramisol on slides coated with $20 \mu \mathrm{l}$ of $0.1 \% \mathrm{w} / \mathrm{v}$ polylysine. Germlines were dissected by removing the heads of animals, using two $27 \mathrm{G}$ needles. $1 \times \mathrm{PBS}+4 \mathrm{mM}$ Tetramisol was replaced with $30 \mu \mathrm{l}$ fresh $2 \%$ paraformaldehyde in $1 \times$ PBS for $10 \mathrm{~min}$ at room temperature. Paraformaldehyde was prepared by dissolving $0.04 \mathrm{~g}$ dry paraformaldehyde in $1 \mathrm{~mL}$ $\mathrm{dH}_{2} 0+2 \mu \mathrm{l} 1 \mathrm{M} \mathrm{NaOH}$ at $65^{\circ} \mathrm{C}$ for $1 \mathrm{~h}$ with occasional mixing, followed by removing $500 \mu \mathrm{l}$ from the tube, and replacing with $500 \mu$ of $\mathrm{H}_{2} \mathrm{PO}_{4}$ buffer $(0.004 \mathrm{~g}$ $\left.\mathrm{KH}_{2} \mathrm{PO}_{4}, 0.0188 \mathrm{~g} \mathrm{NaHPO}_{4}, 500 \mu \mathrm{l} \mathrm{H} \mathrm{O}, \mathrm{pH}=7.2\right)$. After germlines were isolated, coverslips were added to slides and placed on dry ice for at least $5 \mathrm{~min}$, followed by freeze-cracking by rapidly removing the coverslip. Germlines were then immediately fixed by immersing the slides in $100 \%$ methanol for $5 \mathrm{~min}$ and transferred to a 1:1 mixture of methanol:acetone for $5 \mathrm{~min}$. Finally, to end fixation, slides were transferred to $100 \%$ acetone for 5 min. Slides were then washed with $30 \mu \mathrm{l} 1 \times$ PBS $+0.1 \%$ Tween 20 "PBST" for $10 \mathrm{~min}$, three times and incubated with one drop of Image-it added to each slide, for $20 \mathrm{~min}$. Germlines were blocked with $30 \mu \mathrm{l}$ PBST $+1 \%$ BSA for $1 \mathrm{~h}$, followed by overnight incubation with $30 \mu \mathrm{l}$ 1:100 anti-dp-Erk-1 plus 1:100 anti-Nuclear Pore Complex in (PBST + 1\% BSA) (Supplementary Table 2) at room temperature with parafilm over the slide. The next day, parafilm was removed, and slides washed three times with $30 \mu \mathrm{l} 1 \times$ PBST for $10 \mathrm{~min}$. $30 \mu \mathrm{l}$ of secondary 1:500 goat anti-rabbit Alexa 488 for p-Erk1, plus 1:500 donkey antimouse Alexa 568 for Mab414 in (PBST + 1\% BSA) were added to each slide, incubating at room temperature for $1 \mathrm{~h}$. Slides were then washed once with $30 \mu \mathrm{l} 1 \times$ PBST for $10 \mathrm{~min}$ and incubated with $30 \mu \mathrm{l}$ DAPI for $10 \mathrm{~min}(1: 1000$ of $1 \mathrm{mg} / \mathrm{mL}$ DAPI in PBST) followed by a final wash with $30 \mu \mathrm{l} 1 \times$ PBST for $10 \mathrm{~min}$. PBST was removed and $5 \mu \mathrm{l}$ of Prolong Gold was added to each slide with a coverslip and sealed.

EMS mutagenesis and F2 starvation screen. kri-1(ok1251) late L4/young adult worms were incubated with $50 \mathrm{mM}$ EMS (Supplementary Table 3) for $4 \mathrm{~h}$ in $15 \mathrm{~mL}$ conical tubes and allowed to lay eggs on NGM plates. Approximately 500,000 F1 worms (1,000,000 haploid genomes) were divided into 20 separate populations and left to lay F2 eggs. These eggs were transferred to twenty $50 \mathrm{~mL}$ conical tubes with liquid M9 buffer (one tube for each population) and allowed to hatch into L1 larvae. If left under these conditions without food, the larvae arrest growth, and remain in a state of diapause. kri-1(ok1251) F2 larvae were kept in M9 for 1 week, and the entire population from each of the 20 tubes was transferred onto 20 large plates with growth media (one plate for each tube). Since kri-1(ok1251) L1 larvae normally do not survive a week of diapause, only animals with mutations that suppress this phenotype grew to adulthood. These survivors were singled out and clonal lines were established by self-fertilization. F3 progeny from these single survivors were assessed for a restoration of IR-induced apoptosis compared to nonirradiated controls. A single suppressor line was chosen from any given starting population, such that 13 candidates from the 20 populations were sent for whole genome sequencing.

Whole genome sequencing and analysis. DNA was isolated using the DNeasy Blood \& Tissue Kit (Supplementary Table 4) and barcoded libraries were created for each strain using the Nextera DNA Sample Preparation Kit from Illumina, and a HiSeq2500 instrument was used to produce 75 base paired-end reads. First, the sequencing reads were examined for sequence quality using FastQC (http://www. bioinformatics.babraham.ac.uk/projects/fastqc/) to ensure good quality up to the full 75 bases. Bases lower than a quality threshold of 30 were trimmed off using Trimmomatic, however very little trimming was required. Reads were aligned to the C. elegans N2 reference genome (release W220) using BWA-mem. Alignments were sorted by coordinate order and duplicates removed using Picard (http:// picard.sourceforge.net). Before variant calling, reads were processed in Genome Analysis Tool Kit (GATK) v2.5 for indel realignment and base quality score recalibration, using known C. elegans variants from dbSNP build 138 (http://www. ncbi.nlm.nih.gov/SNP/). GATK HaplotypeCaller was used to call variants, and results were filtered for a Phred-scaled $Q$ score $>30$. For each SNP a call is made based on (1) the number of reads, (2) the Phred score of the reads, and (3) how many of each alternate allele is identified. For each SNP an entry was made giving the zygosity-quality $(Q)$ score-\#reads. Finally, called variants were annotated using Annovar to obtain a list of variants for each sample.

Cloning. To generate the Pmpk-2::gfp transcriptional reporter $6 \mathrm{~kb}$ upstream of the $m p k-2$ start codon was amplified from wild type genomic DNA and ligated into the SphI and AgeI restriction sites of the Fire Kit vector pPD95.75. The Pmpk-2::mpk-2 construct was generated by replacing gfp in the above Pmpk-2::gfp plasmid with $m p k-2$ amplified from wild type genomic DNA and ligated into the AgeI and ApaI restriction sites of the above plasmid. The correct sequence of $m p k-2$ was verified by Sanger sequencing. The Pelt-2::mpk-2 construct was generated by ligating $m p k-2$ into the EagI and ApaI restriction sites of pJM559 which contains $5 \mathrm{~kb}$ of the elt- 2 promoter. The Pzipt-2.3::zipt-2.3::gfp translational fusion construct was generated by amplifying Pzipt-2.3::zipt-2.3 starting from $5 \mathrm{~kb}$ upstream of the zipt-2.3 start codon and ending after the $3^{\prime} \mathrm{UTR}$ of zipt-2.3 from wild type genomic DNA. This product was then inserted into BamHI and KpnI restriction sites of the Fire Kit 
vector pPD95.75. The correct sequence of $z i p t-2.3$ was verified by Sanger sequencing. Cloning was conducted using NEB restriction enzymes and buffers, and Invitrogen T4 ligase and buffer. All primers used are listed in Supplementary Table 5 .

Generation of transgenic strains. Constructs were initially injected into wild type $\mathrm{N} 2$ worms at a concentration of $50 \mathrm{ng} / \mu \mathrm{l}$ to create extra-chromosomal arrays. The plasmids pCFJ90 (Pmyo-2:::mcherry) and pCFJ104 (Pmyo-3::mcherry) were used as co-injection markers for Pmpk-2::mpk-2 and Pelt-2::mpk-2 and injected at concentrations of 2.5 and $5 \mathrm{ng} / \mu \mathrm{l}$, respectively.

RNA isolation. 2000 Synchronized L1 worms per condition were grown in biological triplicate and irradiated $24 \mathrm{~h}$ post $\mathrm{L} 4$ and transferred to $1.5 \mathrm{~mL}$ conical tubes using M9 buffer 3-6h post 60 Gy of IR. Worms were washed three times with M9 buffer, M9 removed, and $1 \mathrm{~mL}$ of Trizol added. Worms in Trizol were flash frozen using liquid nitrogen and subjected to three rounds of freeze-thawing. $200 \mu \mathrm{l}$ of chloroform was added, and samples and vortexed for $30 \mathrm{~s}$. Samples were then centrifuged at $12,000 \times g$ for $15 \mathrm{~min}$ at $4^{\circ} \mathrm{C}$. The aqueous phase was transferred to new tubes, and $500 \mu \mathrm{l}$ of $100 \%$ cold isopropanol added to samples and left to precipitate overnight at $-20^{\circ} \mathrm{C}$. The next day, samples were centrifuged at $12,000 \times g$ for $15 \mathrm{~min}$ at $4{ }^{\circ} \mathrm{C}$, and supernatant was removed from the tubes. RNA pellets were washed with $1 \mathrm{~mL}$ of $75 \%$ ethanol in DEPC $\mathrm{dH}_{2} \mathrm{O}$ and vortexed for 1 min. Samples were centrifuged at $12,000 \times g$ for $5 \mathrm{~min}$ at $4^{\circ} \mathrm{C}$ and supernatant discarded. The RNA pellet was air dried for $5 \mathrm{~min}$ and resuspended in $50 \mu \mathrm{l}$ of Invitrogen Ultrapure $\mathrm{dH}_{2} \mathrm{O}$.

RNA sequencing and analysis. mRNA was purified from total RNA by the Hospital for Sick Children Next Generation Sequencing Facility (TCAG) and reads were compared to the wild type samples using Tophat/Cufflinks. Sequencing reads were analyzed for quality with Fastqc. Trimmomatic was used to eliminate lowquality reads and remove adapters. Specific to C. elegans, the minimum intron length in Tophat was lowered to $30 \mathrm{bp}$ from the default 70 due to small intron sizes in C. elegans. The reference genome and transcript annotations were based on release WS235 (CE11).

Quantitative RT-PCR. To determine changes in zipt-2.3 mRNA levels in C. elegans, total RNA was extracted as described above, $3-6 \mathrm{~h}$ post $0 \mathrm{~Gy}$ or $60 \mathrm{~Gy}$. cDNA was prepared from $500 \mathrm{ng}$ of total RNA using the Invitrogen SuperScriptIII FirstStrand Synthesis System with total RNA primed with Random Primer Mix. The reaction was incubated at $25^{\circ} \mathrm{C}$ for $10 \mathrm{~min}, 50^{\circ} \mathrm{C}$ for $60 \mathrm{~min}$, and $85^{\circ} \mathrm{C}$ for $5 \mathrm{~min}$. $1 \mathrm{U}$ of RNaseH was added per sample and incubated at $37^{\circ} \mathrm{C}$ for $30 \mathrm{~min}$. zipt-2.3 transcript levels were quantified in wild type, kri-1(ok1251), kri-1(ok1251); mpk-2 (ok219), mpk-2(ok219), kri-1; klf-3(on34), and klf-3(on34) animals using the BioRad SYBR Green Supermix and the BioRad CFX96 Real-Time PCR Detection System. Transcript levels were normalized to an internal tubulin control, and repeated with two biological replicates. The cDNA was amplified by qPCR using the following conditions: $95^{\circ} \mathrm{C}$ for $30 \mathrm{~s}, 95^{\circ} \mathrm{C}$ for $15 \mathrm{~s}, 60^{\circ} \mathrm{C}$, and repeated for 40 cycles.

Zinc staining with Fluozin-3. $10 \mu \mathrm{M}$ Fluozin-3 was added to NGM plates with OP50 E. coli and left to dry in the dark for $30 \mathrm{~min}$. L4 stage worms were added to the plates and incubated in the dark at $20^{\circ} \mathrm{C}$ for $24 \mathrm{~h}$. Worms were transferred to new NGM plates without dye for $30 \mathrm{~min}$ to de-stain, either irradiated (60 Gy) or left unirradiated, and imaged 3-6 h post IR. To distinguish Fluozin-3 fluorescence from gut granule autofluorescence, wild type worms without dye were imaged.

Zinc staining with Zinpyr-1. $100 \mu \mathrm{M} \mathrm{ZnSO}{ }_{4}$ was added to NGM plates with OP50 E. coli. L4 stage worms were added to the plates and left at $20^{\circ} \mathrm{C}$ for $20 \mathrm{~h} .20 \mu \mathrm{M}$ Zinpyr-1 was added to the plates, and worms incubated in the dark at $20^{\circ} \mathrm{C}$ for $2 \mathrm{~h}$. Worms were transferred to new NGM plates without dye for $30 \mathrm{~min}$ to de-stain and immediately imaged.

ICP- MS. 5000 Synchronized L1 worms were grown in biological triplicate on $\mathrm{NGM}$ and NGM $+0.1 \mathrm{mM} \mathrm{ZnSO}_{4}$ plates. Young adult worms were collected by washing three times in $10 \mathrm{~mL} \mathrm{M} 9+0.1 \%$ Triton $\mathrm{X}-100$. Worms were pelleted in $100 \mu \mathrm{l} \mathrm{M} 9$ buffer and stored at $-80^{\circ} \mathrm{C}$. Worm pellets were then freeze-thawed three times using dry ice with $100 \%$ ethanol and $500 \mu \mathrm{l}$ of Droso buffer $+0.1 \mathrm{mM}$ DTT $+0.1 \%$ NP40. Worm pellets were sonicated in a water bath for 30 cycles $(30 \mathrm{~s}$ on, followed by $30 \mathrm{~s}$ off). $10 \mu \mathrm{l}$ of the worm lysate was used to determine protein concentration using the Bradford assay. $300 \mu \mathrm{l}$ of trace metal grade nitric acid and $100 \mu \mathrm{l}$ of trace metal grade hydrogen peroxide were added to $600 \mu \mathrm{l}$ of worm lysate. Worm lysates were sonicated again for 20 cycles. Finally, the samples were diluted to $2 \%$ nitric acid in a final $15 \mathrm{~mL}$ volume by adding each sample to $14 \mathrm{~mL}$ ultra pure $\mathrm{dH}_{2} \mathrm{O}$. Zinc standards were also prepared. Zinc levels were measured by ICPMS at the University of Toronto (Mississauga campus) Core Instrument Facility.

CRISPR/Cas9 genome editing. Wild type young adult hermaphrodites were injected with crRNA and repair templates for $k l f-3$ (on34) and $d p y-10$ as a marker for positive selection. Dumpy and Roller F1 worms were singled out onto individual plates and screened for the klf-3(on34) mutation by PCR and sequencing. The positive candidate was outcrossed two times to remove the $d p y-10$ mutation. Oligonucleotides used are listed in Supplementary Table 5.

Affinity purification and mass spectrometry. 800,000 wild type, KRI-1::GFP, and ACT-5::GFP expressing strains were each grown in biological triplicate on ten large $90 \mathrm{~mm}$ NGM plates with OP50 E. coli. Young adult worms were either irradiated ( $60 \mathrm{~Gy}$ ) or left unirradiated. Worms were collected $6 \mathrm{~h}$ later by washing with M9 buffer and transferred to a $15 \mathrm{~mL}$ conical tube followed by two more $\mathrm{M} 9$ washes. To remove bacteria from the intestines, worms were incubated and rocked for 30 min in $10 \mathrm{~mL}$ M9 buffer. Afterwards, worms were washed three times more in M9 followed by two washes in autoclaved $\mathrm{dH}_{2} \mathrm{O}$ and pelleted. Worm pellets were flashfrozen in liquid nitrogen and stored at $-80^{\circ} \mathrm{C}$.

To begin GFP affinity purification, worm pellets were thawed on ice in ice-cold DROSO lysis buffer (30 mM HEPES pH 7.4, $100 \mathrm{mM}$ Potassium Acetate, $2 \mathrm{mM}$ Magnesium Acetate, $0.1 \%$ NP-40, 2 mM DTT), with one Mini Protease Inhibitor Cocktail tablet, 1:100 Phosphatase Inhibitor 2, 1:100 Phosphatase Inhibitor 3. Worms were lysed by a dounce homogenization in a chilled Wheaton Dounce Homogenizer (metal) and the lysates were centrifuged at $10,000 \times g$ for $30 \mathrm{~min}$ at $4^{\circ} \mathrm{C}$, and the supernatant transferred to fresh tubes. For affinity purification, $10 \mathrm{mg}$ of protein from each sample was incubated for $3 \mathrm{~h}$ at $4{ }^{\circ} \mathrm{C}$ on a nutator in $30 \mu \mathrm{l}$ of a $50 \%$ slurry of GFP-Trap ${ }^{\circledR} \_$MA beads pre-washed in DROSO buffer. Using a magnetic rack to secure the beads, the supernatant was removed, and the beads were then transferred to a new $1.5 \mathrm{~mL}$ microcentrifuge tube in DROSO buffer. Beads were washed once in TAP buffer (50 mM HEPES-KOH pH 8.0, $100 \mathrm{mM}$ $\mathrm{KCl}, 2 \mathrm{mM}$ EDTA, $0.1 \% \mathrm{NP}-40$, and $10 \%$ glycerol), and once in $50 \mathrm{mM}$ ammonium bicarbonate, $\mathrm{pH}$ 8. Supernatant was removed and beads were resuspended in $10 \mu \mathrm{l}$ of $50 \mathrm{mM}$ ammonium bicarbonate containing $1 \mu \mathrm{g}$ of mass spectrometry-grade trypsin. Samples were digested on beads overnight at $37^{\circ} \mathrm{C}$ on a rotator. The next day, each sample was spun down at $400 \times g$ for $1 \mathrm{~min}$ to pellet beads and the supernatant transferred to a new $1.5 \mathrm{~mL}$ microcentrifuge tube. This supernatant was further digested for $4 \mathrm{~h}$ by supplanting with $0.25 \mu \mathrm{g}$ of trypsin. The digestion was stopped by adding $1 \mu \mathrm{l}$ of $50 \%$ formic acid, and samples were lyophilized and stored at $-80^{\circ} \mathrm{C}$. Samples were then sent for mass spectrometry at the LunenfeldTanenbaum Research Institute in Toronto, Canada.

All proteins were analyzed with Significance Analysis of INTeractome (SAINT) using SAINTexpress (version 3.6.1) to identify true interaction partners using default parameters. Proteins with a probability $\geq 0.95$ were considered, corresponding to an estimated protein level false-discovery rate (FDR) of approximately $0.5 \%$.

Sequence and structure analysis. The sequences of the KRI-1 binding partners identified by mass spectrometry were analyzed by unsupervised threading using Phyre2 (Protein Homology/analogY Recognition Engine v2.0; http://www.sbg.bio ic.ac.uk/phyre2/html/page.cgi?id=index) using the "intensive" model with default options. For each sequence/structure displayed, the model with the highest prediction for each domain was selected, and a complete structural prediction was generated by Phyre2. Starting from K07A9.3, the highest matching template was PDB:4WJ7 "CCM2 PTB domain in complex with KRIT1 NPxY/F3", with a confidence score of 100.0 despite a modest $20 \%$ sequence identity. The second highest matching template was PDB:4FQN "Crystal structure of the CCM2 C-terminal Harmonin Homology Domain (HHD)", with a confidence score of 99.8, and 21\% sequence identity. For Y45F10D.10, the top hit was PDB 4DX9: "ICAP1 in complex with integrin beta 1 cytoplasmic tail", with a confidence score of 100.0 and $25 \%$ sequence identity. Sequence alignments to these structural templates were generated in Phyre2, and manually converted to FASTA ALN for image generation in BoxShade (https://embnet.vital-it.ch/software/BOX_form.html). Structural visualization was in PyMOL (PyMOL Molecular Graphics Systems v 2.0.7)

Transcriptomes of human CCM lesions. Five human CCM lesions were surgically resected for clinical indications unrelated to the research. The specimens were snap frozen in the operating room upon excision, embedded in optimal cutting temperature $(\mathrm{OCT})$, and stored at $-80^{\circ} \mathrm{C}$. Brain tissue from 3 patients free of neurological disease was acquired during autopsy, fixed in formalin and embedded in paraffin blocks (FFPE). Sections of $5 \mu \mathrm{M}$ of the tissue samples were processed, mounted on Leica glass slides (Leica Biosystems, Wetzlar, Germany), and stained for frozen tissue. The endothelial cells (ECs) of CCMs and normal capillaries were then collected using laser capture microdissection (LCM; Leica LMD 6500) at 40× of magnification, and then stored at $-80^{\circ} \mathrm{C}$. The RNAs of the collected endothelial cells were extracted using TRIzol. RNAs from human lesion and control samples were sequenced in 2 batches with 47 basepair (bp) single-end reads. RNA libraries were generated, multiplexed and sequenced on Illumina HiSeq 4000 platform. The quality of raw sequencing reads was then assessed by FastQC (available at http:// www.bioinformatics.babraham.ac.uk/projects/fastqc/). The post-alignment quality control was evaluated using RSeQC and Picard tools. Finally, reads were mapped to UCSC human genome model (hg19) using STAR. Gene transcripts were assembled and quantified based on the corresponding human genome (UCSC RefSeq hg19) using count-based method featureCounts. Differentially expressed genes (DEGs) 
analysis were conducted using DESeq2, with an additive model for batch effect correction when needed. The DEGs were identified at a standard statistically significant threshold of false discovery adjusted $P$-value (FDR) of 0.05 and fold change of 1.2. Human sample collection complied with all relevant ethical regulations. The study protocol was approved by the Institutional Review Board at University of Chicago Medicine, and informed consent was obtained from each subject.

Mouse brain microvascular endothelial cell transcriptomes. Three endothelialspecific conditional Krit1 null mice were generated using a Pdgfb promoter driven tamoxifen-inducible Cre recombinase, Pdgfb-iCreERT2; Krit1fl/fl. Three other wild-type mice were used as controls. BMECs were isolated and maintained at $37^{\circ}$ $\mathrm{C}$ in $5 \% \mathrm{CO}_{2}$ and $95 \%$ air and treated with $5 \mu \mathrm{M}$ of 4 -hydroxytamoxifen for $48 \mathrm{~h}$ to induce allelic loss in BMECs harboring the Cre-recombinase system and floxed alleles. RNAs were extracted using the TRIzol protocol. Illumina's TruSeq Stranded mRNA Sample Prep Kit was used for libraries generation. RNA samples of Krit1 treated mice together with their corresponding null mice were sequenced with 100 bp single-end sequencing reads. The quality of raw sequencing reads was assessed using FastQC v0.11.2 and the post-alignment QC was evaluated with RSeQC and Picard tools vl.117 (http://broadinstitute.github.io/picard/). Reads were mapped to Gencode mouse genome model (GRCm38 V13) using STAR STAR_2.5.3a. Gene transcripts were assembled and quantified on the corresponding mouse genome annotation file using count-based method featureCounts. DEGs analysis were conducted with DESeq2, using an additive model for batch effect correction when needed. DEGs were identified at a standard statistically significant threshold of false discovery corrected $P$-value (FDR) of 0.05 and fold change of 1.2. Gene Ontology enrichment analysis was conducted with $\mathrm{R}$ bioconductor package clusterProfiler based on the GO database on mouse species.

Reporting summary. Further information on experimental design is available in the Nature Research Reporting Summary linked to this article.

\section{Data availability}

All raw mass spectrometry data and SAINTexpress result tables are deposited in the MassIVE repository housed at the Center for Computational Mass Spectrometry at UCSD (https://massive.ucsd.edu/ProteoSAFe/static/massive.jsp). The dataset has been assigned the accession number MSV000082400. The dataset was also contributed to the ProteomeXchange Consortium with identifier PXD009886.

Received: 5 July 2018 Accepted: 2 April 2019

Published online: 17 April 2019

\section{References}

1. Elmore, S. Apoptosis: a review of programmed cell death. Toxicol. Pathol. 35, 495-516 (2007).

2. Horvitz, H. R. Genetic control of programmed cell death in the nematode Caenorhabditis elegans. Cancer Res. 59, 1701s-1706s (1999).

3. Gartner, A., Milstein, S., Ahmed, S., Hodgkin, J. \& Hengartner, M. O. A conserved checkpoint pathway mediates DNA damage-induced apoptosis and cell cycle arrest in C. elegans. Mol. Cell 5, 435-443 (2000).

4. Gumienny, T. L., Lambie, E., Hartwieg, E., Horvitz, H. R. \& Hengartner, M. O. Genetic control of programmed cell death in the Caenorhabditis elegans hermaphrodite germline. Development 126, 1011-1022 (1999).

5. Nehme, R. \& Conradt, B. egl-1: a key activator of apoptotic cell death in $C$. elegans. Oncogene 27, S30-S40 (2008).

6. Derry, W. B. Caenorhabditis elegans p53: role in apoptosis, meiosis, and stress resistance. Science 294, 591-595 (2001).

7. Schumacher, B., Hofmann, K., Boulton, S. \& Gartner, A. The C. elegans homolog of the p53 tumor suppressor is required for DNA damage-induced apoptosis. Curr. Biol. 11, 1722-1727 (2001).

8. Hofmann, E. R. et al. Caenorhabditis elegans HUS-1 is a DNA damage checkpoint protein required for genome stability and EGL-1-mediated apoptosis. Curr. Biol. 12, 1908-1918 (2002).

9. Rutkowski, R. et al. Regulation of Caenorhabditis elegans p53/CEP-1dependent germ cell apoptosis by Ras/MAPK signaling. PLoS Genet. 7, e1002238 (2011).

10. Eberhard, R. et al. Ribosome synthesis and MAPK activity modulate ionizing radiation-induced germ cell apoptosis in Caenorhabditis elegans. PLoS Genet. 9, e1003943 (2013).

11. Perrin, A. J. et al. Noncanonical control of C. elegans germline apoptosis by the insulin/IGF-1 and Ras/MAPK signaling pathways. Cell Death Differ. 20, 97-107 (2013).

12. Ito, S., Greiss, S., Gartner, A. \& Derry, W. B. Cell-nonautonomous regulation of C. elegans germ cell death by kri-1. Curr. Biol. 20, 333-338 (2010).
13. Morrison, L. \& Akers, A. Cerebral cavernous malformation, familial. GeneReviews (2003).

14. Berman, J. R. \& Kenyon, C. Germ-cell loss extends C. elegans life span through regulation of DAF-16 by kri-1 and lipophilic-hormone signaling. Cell 124, 1055-1068 (2006)

15. Renz, M. et al. Regulation of $\beta 1$ integrin-Klf2-mediated angiogenesis by CCM proteins. Dev. Cell 32, 181-190 (2015).

16. Zhou, Z. et al. The cerebral cavernous malformation pathway controls cardiac development via regulation of endocardial MEKK3 signaling and KLF expression. Dev. Cell 32, 168-180 (2015).

17. Akers, A. et al. Synopsis of guidelines for the clinical management of cerebral cavernous malformations: consensus recommendations based on systematic literature review by the Angioma Alliance Scientific Advisory Board Clinical Experts Panel. Neurosurgery 80, 665-680 (2017)

18. Lee, M. H. et al. Multiple functions and dynamic activation of MPK-1 extracellular signal-regulated kinase signaling in Caenorhabditis elegans germline development. Genetics 177, 2039-2062 (2007).

19. Kritikou, E. A. et al. C. elegans GLA-3 is a novel component of the MAP kinase MPK-1 signaling pathway required for germ cell survival. Genes Dev. 20, 2279-2292 (2006).

20. Eisenmann, D. M. \& Kim, S. K. Mechanism of activation of the Caenorhabditis elegans ras homologue let- 60 by a novel, temperaturesensitive, gain-of-function mutation. Genetics 146, 553-565 (1997).

21. Drew, B. A., Burow, M. E. \& Beckman, B. S. MEK5/ERK5 pathway: the first fifteen years. Biochim. Biophys. Acta Rev. Cancer. https://doi.org/10.1016/j. bbcan.2011.10.002 (2012).

22. McConnell, B. B. \& Yang, V. W. Mammalian Krüppel-like factors in health and diseases. Physiol. Rev. 90, 1337-1381 (2010).

23. Hodgkin, J. Karyotype, ploidy, and gene dosage. WormBook. https://doi.org/ 10.1895/wormbook.1.3.1 (2005).

24. Zhou, Z. et al. Cerebral cavernous malformations arise from endothelial gain of MEKK3-KLF2/4 signalling. Nature 532, 122-126 (2016).

25. Stinchcomb, D. T., Shaw, J. E., Carr, S. H. \& Hirsh, D. Extrachromosomal DNA transformation of Caenorhabditis elegans. Mol. Cell. Biol. 5, 3484-3496 (1985).

26. Kelly, W. G., Xu, S., Montgomery, M. K. \& Fire, A. Distinct requirements for somatic and germline expression of a generally expressed Caenorhabditis elegans gene. Genetics 146, 227-238 (1997).

27. Uhlik, M. T. et al. Rac-MEKK3-MKK3 scaffolding for p38 MAPK activation during hyperosmotic shock. Nat. Cell Biol. 5, 1104-1110 (2003).

28. Fisher, O. S. et al. Structure and vascular function of MEKK3-cerebral cavernous malformations 2 complex. Nat. Commun. 6, 7937 (2015).

29. Zhang, H. et al. Clathrin and AP-1 regulate apical polarity and lumen formation during C. elegans tubulogenesis. Development 139, 2071-2083 (2012).

30. Zhang, J., Clatterbuck, R., Rigamonti, D., Chang, D. \& Dietz, H. Interaction between krit1 and icapla infers perturbation of integrin $\beta 1$-mediated angiogenesis in the pathogenesis of cerebral cavernous malformation. Hum. Mol. Genet. 10, 2953-2960 (2001).

31. Liu, W., Draheim, K. M., Zhang, R., Calderwood, D. A. \& Boggon, T. J. Mechanism for KRIT1 release of ICAP1-mediated suppression of integrin activation. Mol. Cell. https://doi.org/10.1016/j.molcel.2012.12.005 (2013).

32. Fisher, O. S. et al. Structural basis for the disruption of the Cerebral Cavernous Malformations 2 (CCM2) interaction with Krev Interaction Trapped 1 (KRIT1) by disease-associated mutations. J. Biol. Chem. 290, 2842-2853 (2015).

33. Fisher, O. S. et al. Structural studies of cerebral cavernous malformations 2 (CCM2) reveal a folded helical domain at its C-terminus. FEBS Lett. https:// doi.org/10.1016/j.febslet.2012.12.011 (2013).

34. Zawistowski, J. S. et al. CCM1 and CCM2 protein interactions in cell signaling: Implications for cerebral cavernous malformations pathogenesis. Hum. Mol. Genet. 14, 2521-2531 (2005).

35. Ganju, N. \& Eastman, A. Zinc inhibits Bax and Bak activation and cytochrome $c$ release induced by chemical inducers of apoptosis but not by death-receptorinitiated pathways. Cell Death Differ. 10, 652-661 (2003).

36. Perry, D. K. et al. Zinc is a potent inhibitor of the apoptotic protease, caspase3: a novel target for zinc in the inhibition of apoptosis. J. Biol. Chem. 272, 18530-18533 (1997).

37. Roh, H. C., Collier, S., Guthrie, J., Robertson, J. D. \& Kornfeld, K. Lysosomerelated organelles in intestinal cells are a zinc storage site in C. elegans. Cell Metab. 15, 88-99 (2012).

38. Roh, H. C. et al. ttm-1 encodes CDF transporters that excrete zinc from intestinal cells of $C$. elegans and act in a parallel negative feedback circuit that promotes homeostasis. PLoS Genet. 9, el003522 (2013).

39. Dietrich, N., Schneider, D. L. \& Kornfeld, K. A pathway for low zinc homeostasis that is conserved in animals and acts in parallel to the pathway for high zinc homeostasis. Nucleic Acids Res. https://doi.org/10.1093/nar/ gkx762 (2017) 
40. Davis, D. E. et al. The cation diffusion facilitator gene cdf-2 mediates zinc metabolism in Caenorhabditis elegans. Genetics 182, 1015-1033 (2009).

41. Schroeder, L. K. et al. Function of the Caenorhabditis elegans ABC transporter PGP-2 in the biogenesis of a lysosome-related fat storage organelle. Mol. Biol. Cell 18, 995-1008 (2007).

42. Dietrich, N., Tan, C. H., Cubillas, C., Earley, B. J. \& Kornfeld, K. Insights into zinc and cadmium biology in the nematode Caenorhabditis elegans. Arch. Biochem. Biophys. https://doi.org/10.1016/j.abb.2016.05.021 (2016).

43. Zhao, Y. et al. The zinc transporter ZIPT-7.1 regulates sperm activation in nematodes. PLoS Biol. https://doi.org/10.1371/journal.pbio.2005069 (2018).

44. Hester, J., Hanna-Rose, W. \& Diaz, F. Zinc deficiency reduces fertility in C elegans hermaphrodites and disrupts oogenesis and meiotic progression. Comp. Biochem. Physiol. C Toxicol. Pharmacol. https://doi.org/10.1016/j. cbpc.2016.09.006 (2017).

45. Spector, M. S., Desnoyers, S., Hoeppner, D. J. \& Hengartner, M. O. Interaction between the C. elegans cell-death regulators CED-9 and CED- 4. Nature 385, 653-656 (1997).

46. Lopez-Ramirez, M. A. et al. Thrombospondin1 (TSP1) replacement prevents cerebral cavernous malformations. J. Exp. Med. https://doi.org/10.1084/ jem.20171178 (2017)

47. Cullere, X., Plovie, E., Bennett, P. M., MacRae, C. A. \& Mayadas, T. N. The cerebral cavernous malformation proteins CCM2L and CCM2 prevent the activation of the MAP kinase MEKK3. Proc. Natl. Acad. Sci. U.S.A. 112, 14284-14289 (2015).

48. Hayashi, M. et al. Targeted deletion of BMK1/ERK5 in adult mice perturbs vascular integrity and leads to endothelial failure. J. Clin. Invest. 113, $1138-1148$ (2004).

49. Im, J. Y. et al. DNA damage induced apoptosis suppressor (DDIAS) is upregulated via ERK5/MEF2B signaling and promotes $\beta$-catenin-mediated invasion. Biochim. Biophys. Acta Gene Regul. Mech. 1859, 1449-1458 (2016).

50. Sunadome, K. et al. ERK5 regulates muscle cell fusion through Klf transcription factors. Dev. Cell 20, 192-205 (2011).

51. Zhang, J. et al. Mutation in Caenorhabditis elegans Kruppel-like factor, KLF-3 results in fat accumulation and alters fatty acid composition. Exp. Cell Res. 315, 2568-2580 (2009).

52. Franklin, R. B. \& Costello, L. C. The important role of the apoptotic effects of zinc in the development of cancers. J. Cell. Biochem. 106, 750-757 (2009).

53. Maret, W. Zinc in cellular regulation: the nature and significance of "zinc signals”. Int. J. Mol. Sci. https://doi.org/10.3390/ijms18112285 (2017).

54. Wang, D., Shen, L. \& Wang, Y. The phenotypic and behavioral defects can be transferred from zinc-exposed nematodes to their progeny. Environ. Toxicol. Pharmacol. https://doi.org/10.1016/j.etap.2007.05.009 (2007).

55. Murphy, J. T. et al. Histidine protects against zinc and nickel toxicity in Caenorhabditis elegans. PLoS Genet. https://doi.org/10.1371/journal. pgen.1002013 (2011).

56. Kumar, J. et al. Zinc levels modulate lifespan through multiple longevity pathways in Caenorhabditis elegans. PLoS One. https://doi.org/10.1371/ journal.pone.0153513 (2016).

57. Dewson, G. \& Kluck, R. M. Mechanisms by which Bak and Bax permeabilise mitochondria during apoptosis. J. Cell Sci. https://doi.org/10.1242/jcs.038166 (2009).

58. Bruinsma, J. J., Jirakulaporn, T., Muslin, A. J. \& Kornfeld, K. Zinc ions and cation diffusion facilitator proteins regulate ras-mediated signaling. Dev. Cell 2, 567-578 (2002).

59. Yoder, J. H., Chong, H., Guan, K. I. \& Han, M. Modulation of KSR activity in Caenorhabditis elegans by $\mathrm{Zn}$ ions, PAR-1 kinase and PP2A phosphatase. EMBO J. 23, 111-119 (2004)

60. Jirakulaporn, T. \& Muslin, A. J. Cation diffusion facilitator proteins modulate Raf-1 activity. J. Biol. Chem. 279, 27807-27815 (2004).

61. Sarasamma, S. et al. Zinc chloride exposure inhibits brain acetylcholine levels, produces neurotoxic signatures, and diminishes memory and motor activities in adult zebrafish. Int. J. Mol. Sci. https://doi.org/10.3390/ijms19103195 (2018).

62. Yu, X. \& Li, Y. V. Neuroprotective effect of zinc chelator DEDTC in a Zebrafish (Danio rerio) model of hypoxic brain injury. Zebrafish. https://doi. org/10.1089/zeb.2012.0777 (2013).
63. Lin, W. et al. Zinc transporter Slc39a8 is essential for cardiac ventricular compaction. J. Clin. Invest. 128, 826-833 (2018)

\section{Acknowledgements}

The authors are grateful to Dr. Peter Roy for advice on the project, Dr. Abigail Mateo for helping to design Fig. 7, and members of the Derry lab for comments on the manuscript This work was supported by an operating grant from the Canadian Institutes of Health Research (CIHR) to W.B.D. (MOP 137089). A.-C.G. holds a Tier 1 Canada Research Chair in "Functional Proteomics" and was supported by a CIHR Foundation grant (FDN 143301). I.A.A. was supported by an operating grant from the National Institutes of Health (P01 NS092521). S.A.-S. was supported by the Excellence cluster REBIRTH (SFB958) by Deutsche Forschungsgemeinschaft (DFG) projects SE2016/7-2 and SE2016/ 10-1 and by the DZHK. E.M.C. was supported by an Alexander Graham Bell Canada Graduate Scholarship from the Natural Sciences and Engineering Council of Canada and a Terry Fox Foundation Strategic Training in Transdisciplinary Radiation Science Scholarship. R.G. was supported by a Safadi Translational Fellowship and J.K. was supported by the Sigrid Juselius Foundation.

\section{Author contributions}

E.M.C. designed the experiments, performed experiments, analyzed the data, and wrote the manuscript. B.L. performed apoptosis experiments. Y.O. performed cloning and apoptosis experiments and B.Y. performed CRISPR gene editing and zinc quantification. M.S. and A.G.F. assembled and mapped whole genome sequencing reads of suppressor strains. D.D. and S.A.-S. performed zebrafish zinc measurements. J.K., R.G., Y.L., and I.A. A. performed and analyzed RNA sequencing experiments in mouse and patient samples. C.G. and A.-C.G. performed proteomics and structure threading experiments. W.B.D. conceived of the study, performed apoptosis experiments, and helped write the manuscript. All authors reviewed the manuscript before publication.

\section{Additional information}

Supplementary Information accompanies this paper at https://doi.org/10.1038/s41467019-09829-z.

Competing interests: The authors declare no competing interests.

Reprints and permission information is available online at http://npg.nature.com/ reprintsandpermissions/

Journal peer review information: Nature Communications would like to thank David Killilea, Angeliki Louvi, and other anonymous reviewers for their contributions to the peer review of this work. Peer review reports are available.

Publisher's note: Springer Nature remains neutral with regard to jurisdictional claims in published maps and institutional affiliations.

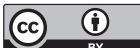

Open Access This article is licensed under a Creative Commons Attribution 4.0 International License, which permits use, sharing, adaptation, distribution and reproduction in any medium or format, as long as you give appropriate credit to the original author(s) and the source, provide a link to the Creative Commons license, and indicate if changes were made. The images or other third party material in this article are included in the article's Creative Commons license, unless indicated otherwise in a credit line to the material. If material is not included in the article's Creative Commons license and your intended use is not permitted by statutory regulation or exceeds the permitted use, you will need to obtain permission directly from the copyright holder. To view a copy of this license, visit http://creativecommons.org/ licenses/by/4.0/.

(c) The Author(s) 2019 